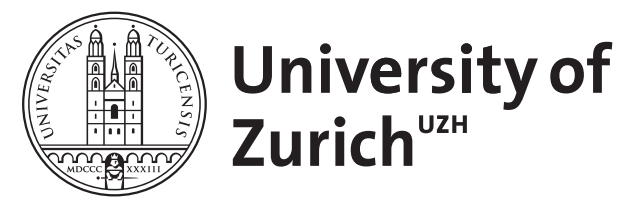

\title{
Optimal taxation with rent-seeking
}

\author{
Rothschild, Casey ; Scheuer, Florian
}

\begin{abstract}
We develop a framework for optimal taxation when agents can earn their income both in traditional activities, where private and social products coincide, and in rent-seeking activities, where private returns exceed social returns either because they involve the capture of pre-existing rents or because they reduce the returns to traditional work. We characterize Pareto optimal income taxes that do not condition on how much of an individual's income is earned in each of the two activities. These optimal taxes feature an externality-corrective term, the magnitude of which depends both on the Pigouvian correction that would obtain if rent-seeking incomes could be perfectly targeted and on the relative impact of rent-seeking externalities on the private returns to traditional and to rent-seeking activities. If rent-seeking externalities primarily affect other rent-seekers, for example, the optimal correction lies strictly below the Pigouvian correction. A calibrated model indicates that the gap between the Pigouvian and optimal correction can be quantitatively important. Our results thus point to a hefty informational requirement for correcting rent-seeking externalities through the income tax code.
\end{abstract}

DOI: https://doi.org/10.1093/restud/rdw017

Posted at the Zurich Open Repository and Archive, University of Zurich ZORA URL: https://doi.org/10.5167/uzh-138643

Journal Article

Accepted Version

Originally published at:

Rothschild, Casey; Scheuer, Florian (2016). Optimal taxation with rent-seeking. Review of Economic Studies, 83(3):1225-1262.

DOI: https://doi.org/10.1093/restud/rdw017 


\title{
Optimal Taxation with Rent-Seeking*
}

\author{
Casey Rothschild \\ Wellesley College
}

\author{
Florian Scheuer \\ Stanford University
}

December 2015

\begin{abstract}
We develop a framework for optimal taxation when agents can earn their income both in traditional activities, where private and social products coincide, and in rentseeking activities, where private returns exceed social returns either because they involve the capture of pre-existing rents or because they reduce the returns to traditional work. We characterize Pareto optimal income taxes that do not condition on how much of an individual's income is earned in each of the two activities. These optimal taxes feature an externality-corrective term, the magnitude of which depends both on the Pigouvian correction that would obtain if rent-seeking incomes could be perfectly targeted and on the relative impact of rent-seeking externalities on the private returns to traditional and to rent-seeking activities. If rent-seeking externalities primarily affect other rent-seekers, for example, the optimal correction lies strictly below the Pigouvian correction. A calibrated model indicates that the gap between the Pigouvian and optimal correction can be quantitatively important. Our results thus point to a hefty informational requirement for correcting rent-seeking externalities through the income tax code.
\end{abstract}

\footnotetext{
*Email addresses: crothsch@wellesley.edu, scheuer@stanford.edu. We are especially grateful to Iván Werning and two anonymous referees for numerous valuable suggestions. We also thank Daron Acemoglu, Alan Auerbach, Marco Bassetto, Michael Boskin, Paco Buera, V. V. Chari, Peter Diamond, Emmanuel Farhi, Mikhail Golosov, Martin Hellwig, Caroline Hoxby, Patrick Kehoe, Phillip Levine, Guido Menzio, Chris Phelan, Thomas Piketty, James Poterba, Emmanuel Saez, Daniel Sichel, Matthew Weinzierl, David Wildasin and seminar participants at Alberta, Berkeley, MPI Bonn, Cologne, Copenhagen, Cornell, Frankfurt, Guelph, the Federal Reserve Bank of Minneapolis, Harvard, Konstanz, Mannheim, Munich, MIT, Stanford, Stockholm School of Economics, UMass-Amherst, Uppsala, Wellesley, Wharton, ETH Zurich, the SED Annual Meeting, NBER Summer Institute and Public Economics Program Meeting, Minnesota Workshop in Macroeconomic Theory, NTA Annual Conference on Taxation, the ASSA Meetings and the Young Macroeconomists' Jamboree (Duke) for helpful comments. Scheuer thanks Harvard University and UC Berkeley and Rothschild thanks the Radcliffe Institute for Advanced Study at Harvard University for hospitality and funding. All errors are our own.
} 


\section{Introduction}

The financial crisis exposed prominent examples of highly compensated individuals whose apparent contributions to social output proved illusory. The view that some top incomes reflect rent-seeking-i.e., the pursuit of personal enrichment by extracting a slice of the existing economic pie rather than by increasing the size of that pie-has inspired calls for a more steeply progressive tax code (Piketty et al., 2014), and, motivated by similar concerns about rent-seeking in finance, various countries have proposed higher taxes on financial-sector bonus payments (Besley and Ghatak, 2013).

The argument behind such proposals is intuitively appealing. If part of the economic activity at high incomes is socially unproductive rent-seeking or "skimming," then it would seem natural for a well-designed income tax to impose high marginal rates at high incomes. ${ }^{1}$ This would discourage such behavior while simultaneously raising revenue that could be used, for instance, to reduce taxes and encourage more productive effort at lower incomes. Moreover, if some sectors or professions are more prone to rent-seeking than others (Lockwood et al., 2015), sector-specific corrective taxes would be useful.

In this paper, we study the optimal design of such policies under the assumption of imperfect targeting. For example, lawyers produce many socially efficient services, upholding property rights and providing incentives to abide by useful rules. On the other hand, they may also engage in rent-seeking activities, some of which resemble zero-sum games. The friction that we account for here is that it can be very hard to tell which is which: the only way to find out can be a costly trial, a highly imperfect process. A similar point can be made of finance and many other sectors. Hence, even sector- or profession-specific taxes are necessarily imperfectly targeted, as they apply to multiple different activities within such sectors that all come together in the same market and cannot be easily disentangled in any given transaction. At the extreme, an individual may engage both in productive and rent-seeking activities simultaneously, but only total income is observable when computing tax liabilities.

Towards cleanly identifying the key effects of rent-seeking on optimal income taxes, we begin with a simple representative-agent Ramsey model which isolates the corrective role for taxation-as there is no scope for redistribution. The identical individuals in this model can pursue two types of activities: traditional, productive work, where private and social returns to effort coincide, and rent-seeking, where private returns exceed the social returns to effort. Suppose, for instance, that rent-seeking effort involves claiming credit for productive work done by others. Then rent-seeking imposes across-activity

\footnotetext{
${ }^{1}$ See Bertrand and Mullainathan (2001) for evidence of such rents.
} 
externalities (i.e., reduces the productivity of traditional effort) as well as within-activity externalities (due to crowding effects in claiming credit). Both externalities drive a wedge between the private returns to effort and its true productivity at the aggregate level.

A natural guess for the optimal tax rate would be the weighted Pigouvian tax rate, i.e., the proportion of income earned through rent-seeking in the economy multiplied by the wedge between the private and social returns to rent-seeking effort-the wedge that would be the optimal tax on rent-seeking if it could be separately identified. Our first main result, however, is that the optimal income tax systematically diverges from this benchmark (Proposition 1).

To see why the initial guess is generally incorrect, consider the effects of a small tax increase. It discourages effort and thus directly reduces rent-seeking. But it also has indirect effects since a reduction in rent-seeking effort raises the returns to both types of effort. If the within-activity externalities are large relative to the across-activity ones, the returns to rent-seeking rise by more than the returns to productive effort. The tax change thus encourages a perverse shift of effort into rent-seeking. This indirect effect partially offsets the direct corrective benefits of the higher tax, and the optimal correction lies strictly below the Pigouvian benchmark. When, on the other hand, the across-activity externalities from rent-seeking dominate the within-activity ones, a reduction in rentseeking effort lowers the relative returns to rent-seeking, the activity shift effect reverses sign, and the optimal correction exceeds the Pigouvian tax rate.

Indeed, we provide a simple and intuitive formula for the optimal correction, which reveals that the gap between the optimal and Pigouvain corrections depend on the product of two key parameters: the elasticity of the relative returns with respect to rent-seeking effort, and individuals' substitution elasticity between the two activities. The former determines the extent to which an additional unit of rent-seeking effort increases or decreases the relative returns to rent-seeking, and the second determines the magnitude of the activity shift in response to a change in relative returns. Only in the knife-edge case where one of these elasticities is zero does the standard Pigouvian correction apply. Conversely, we show that the divergence between the Pigouvian and the optimal correction explodes in this framework as the substitution elasticity grows (so individuals tend to specialize in one of the two activities): The optimal tax can be zero even though rentseeking accounts for a strictly positive share of income in the economy and even though it has strictly negative externalities.

We then extend our analysis to allow for heterogeneity, which is required both to address the question of the optimal progressivity of the income tax schedule, and also to see how the corrective motives interact with the standard redistributive motives for taxation. 
This extension assumes that individuals differ in their skills for both activities and considers the design of a fully nonlinear income tax. We solve this multidimensional screening model by building on the insights in Rothschild and Scheuer (2013). Specifically, we observe that the realized wage distribution depends on the aggregate rent-seeking effort, so that the optimal tax problem can be treated as a fixed point problem for rent-seeking effort nested within an almost-standard Mirrlees (1971) optimal tax problem. The bottom line is that the key intuition from the Ramsey model carries over, but the activity shift effect gets complemented by several additional effects, which are due to heterogeneity.

In particular, our Proposition 2 provides an optimal marginal tax rate formula for each income level. This formula features a multiplicative correction to a standard optimal tax formula for economies without rent-seeking (such as Saez, 2001). This structure is consistent with the "principle of targeting" (Dixit, 1985) and, more specifically, the "additivity principle" discussed in the literature on corrective taxation in the presence of atmospheric externalities, according to which taxes can be expressed as a sum of the optimal Pigouvian taxes and the optimal taxes from a related problem without externalities. ${ }^{2}$ However, Proposition 3 shows that the correction term in the optimal tax formula again diverges from the Pigouvian benchmark in manner that depends in a transparent way on the sign and magnitude of the relative return elasticity.

Interestingly, we find an even stronger divergence result (vis-à-vis the Ramsey model) in this heterogeneous agent model. The reason is that changes in marginal tax rates at any given income level affect aggregate returns and therefore induce activity shifts elsewhere in the income distribution. As a result, the optimal correction diverges from the Pigouvian benchmark even at points in the distribution where all income is from rent-seeking, and where the (marginal) income tax might appear to be a perfectly targeted instrument.

Finally, we complement these analytical results with a quantitative exploration of this divergence. We estimate a flexible bivariate Pareto-lognormal parametrization of the the two-dimensional skill distribution using data from the 2014 Current Population Survey (CPS) and borrow estimates for the externalities from rent-seeking, which we associate with law and finance, from the literature. We then trace out the full set of possible relative return effects, consistent with the given Pigouvian correction, and simulate the optimal nonlinear income tax schedule for each of these scenarios. Our results suggest that the general equilibrium effects we emphasize here can be of similar magnitude as the Pigouvian taxes themselves, leading to considerable divergences.

In summary, our theoretical and quantitative results point to the hefty informational requirements for using the income tax code to correct rent-seeking externalities. Even

\footnotetext{
${ }^{2}$ See Sandmo (1975), Sadka (1978), Cremer et al. (1998), Kopczuk (2003).
} 
if, for example, policymakers could perfectly pin down the overall magnitude of rentseeking externalities, either in the economy overall or in a particular industry such as finance (which is already challenging), our results suggest that there would still be a wide range of possibly optimal corrective taxes. To determine the appropriate corrective tax, policymakers would additionally need to know the relative impact of rent-seeking on various activities. In the absence of clear evidence on this, our results can be interpreted as advising caution in using the income tax as a tool to discourage rent-seeking.

Related Literature. Our main results are most closely related in spirit to Diamond (1973), although our motivation and framework are very different. Most importantly, Diamond analyses the linear taxation of an externality-producing consumption good with heterogeneous agents. We establish our results in a homogenous agent Ramsey setting and then extend them to a Mirrleesian framework with non-linear income taxes, thereby incorporating both corrective and redistributive motives for taxation. Moreover, as we discuss in detail in the following section, our formula characterizing the divergence between the optimal and Pigouvian corrections in the Ramsey model is related to some of Diamond's formulas, but in contrast to them our formula shows that this divergence depends in an intuitive way on the product of two simple elasticities with natural empirical analogs, allowing us to get transparent results on its direction and magnitude.

A special case of our model obtains when rent-seeking income is earned through a crowdable search activity. Our analysis is therefore related to recent work by Golosov et al. (2013), who consider optimal taxation in labor markets with search frictions. However, they abstract from skill-driven wage heterogeneity in contrast to the general heterogeneity we allow for. Moreover, they consider search for employment rather than search $a$ an income producing (but, through crowding, negative externality generating) activity (see also Hungerbuhler et al., 2006).

Our analysis tracks the methods of the optimal income taxation literature, notably Ramsey (1927), Mirrlees (1971), Diamond (1998), Saez (2001) and Werning (2007). Our paper also contributes to recent efforts to study optimal taxation under multidimensional private heterogeneity-a literature which needs to tackle the challenges implied by multidimensional screening problems, as standard techniques typically do not apply (Rochet and Choné, 1998). This literature includes, for instance, Kleven et al. (2009), Scheuer $(2013,2014)$, Beaudry et al. (2009), Choné and Laroque (2010), and Lockwood and Weinzierl (2015). These papers have different information structures than ours, however: the second dimension of heterogeneity enters preferences additively in the first three; in Beaudry et al. (2009), types have two distinct labor productivities, but one activity is a 
non-market activity, the returns from which are unobservable, whereas total incomebut not its breakdown between the two activities-is observable in our model; ${ }^{3}$ and in Choné and Laroque (2010) and Lockwood and Weinzierl (2015), the second dimension is a taste for labor rather than a full second skill type as we employ here.

More closely related is Rothschild and Scheuer (2013), who use methods similar to those developed here to characterize optimal taxation in a Roy (1951) model. That paper shares the structure of two-dimensional heterogeneity in our Mirrlees extension, but (as all the other papers above) considers the special case where individuals always specialize in one type of activity. It also rules out wages that deviate from the social marginal product of effort and the resulting corrective motives for taxation, issues we focus on here. ${ }^{4}$

Finally, our paper relates to the literature studying the equilibrium allocation of talent across different sectors when there are rents to be captured in some of them. Most of this literature (e.g. Baumol, 1990, Murphy et al., 1991, Acemoglu and Verdier, 1998, and Cahuc and Challe, 2012) does not consider optimal tax policy to correct these equilibrium outcomes. But there are important recent exceptions. Philippon (2010) considers an endogenous growth model with financiers, workers and entrepreneurs and analyzes the effect of linear, sector-specific taxes on growth. The recent studies by Piketty et al. (2014) and Lockwood et al. (2015) focus on the case where externalities reduce everyone else's income in a lump-sum fashion rather than the proportional reduction that we consider here. This rules out the relative return effects from effort that we emphasize, which arise when the externalities can affect different activities to varying degrees. In this case, due to the absence of general equilibrium effects, the simple weighted Pigouvian correction is optimal, which we use as a benchmark to compare our results to, both analytically and quantitatively. Furthermore, Lockwood et al. abstract from redistributive motives and Piketty et al. restrict attention to top marginal tax rates.

This paper proceeds as follows. In Section 2, we begin with a representative agent Ramsey model that illustrates the key force underlying our main divergence result. Section 3 then incorporates rich heterogeneity into our modeling framework and discusses tax implementation issues. In Section 4, we analyze this model and provide our main results. Finally, Section 5 provides a quantification of these results for a calibrated version of our model, and Section 6 concludes. Most proofs as well as details on the data and calibration appear in a technical appendix. We also collect various examples and extensions

\footnotetext{
${ }^{3}$ Beaudry et al. also assume that, unlike here, effort in the market activity is observable.

${ }^{4}$ Our Ramsey setting with two unobservable margins of effort also relates to the literature on multitasking (Holmström and Milgrom, 1991 and Baker, 1992), although these papers do not consider externalities and corrective taxation. In Rothschild and Scheuer (2014), we extend our Mirrleesian analysis to $N>2$ activities with arbitrary positive and negative externalities.
} 
in an online appendix.

\section{A Simple Model Without Heterogeneity}

We begin with a simple Ramsey representative agent model, which illustrates the key force underlying our results. We will later show the additional effects that emerge in a rich Mirrlees model with more realistic heterogeneity.

\subsection{Setup}

Preferences. Consider an economy consisting of a unit mass of identical agents, indexed by $i \in[0,1]$. Preferences over consumption $c$ and effort in each of two distinct activities, $e_{\theta}$ and $e_{\varphi}$, are given by $U\left(c, e_{\theta}, e_{\varphi}\right)=u\left(c, m\left(e_{\theta}, e_{\varphi}\right)\right) \equiv u(c, l)$. We associate $\theta$ with a traditional activity and $\varphi$ with rent-seeking, as explained below. We assume that $u$ is strictly quasiconcave, twice continuously differentiable, and has $u_{c}>0$ and $u_{l}<0$. We also impose the regularity conditions that consumption $c$ and "leisure" $-l$ are normal goods and $\lim _{l, c \rightarrow 0}-u_{l} / u_{c}=0$. For concreteness, we consider here the CES-specification for the effort aggregator

$$
m\left(e_{\theta}, e_{\varphi}\right)=\left(e_{\theta}^{\frac{1+\sigma}{\sigma}}+e_{\varphi}^{\frac{1+\sigma}{\sigma}}\right)^{\frac{\sigma}{1+\sigma}}
$$

with constant substitution elasticity $\sigma>0$, but we show in Appendix A that our results go through for more general specifications. For $\sigma \rightarrow 0$, individuals always choose $e_{\theta}=e_{\varphi}$, whereas for $\sigma \rightarrow \infty$, they always specialize in one of the two activities.

Technology. Agents earn incomes $y$ in proportion to each of the two components of their effort via $y=r_{\theta}(E) e_{\theta}+r_{\varphi}(E) e_{\varphi}$, where the activity-specific returns $r_{\theta}(E)=k_{\theta} E^{-\beta_{\theta}}$ and $r_{\varphi}(E)=k_{\varphi} E^{-\beta_{\varphi}}$, with elasticities $\beta_{\theta}, \beta_{\varphi} \in[0,1)$, are decreasing in the aggregate rentseeking effort $E \equiv \int_{0}^{1} e_{\varphi}(i) d i$, and where $k_{\theta}, k_{\varphi}>0$ are some constants. ${ }^{5}$ For later use, denote the elasticity of the relative return $r_{\theta}(E) / r_{\varphi}(E)$ with respect to $E$ by $\Delta \equiv \beta_{\varphi}-\beta_{\theta}$. We also impose the regularity condition $\Delta \sigma>-1+\beta_{\theta}$, which we discuss further below.

Summing over individuals, aggregate income is therefore

$$
Y\left(E_{\theta}, E\right)=r_{\theta}(E) E_{\theta}+r_{\varphi}(E) E,
$$

where $E_{\theta} \equiv \int_{0}^{1} e_{\theta}(i) d i$. It is a sum of two components: the total earnings $Y_{\varphi} \equiv r_{\varphi}(E) E$

\footnotetext{
${ }^{5}$ Appendix A again shows that our results generalize to arbitrary non-increasing return functions, whose elasticities with respect to $E$ are not constant, but depend on $E$.
} 
accruing to rent-seeking, and the total earnings $Y_{\theta} \equiv r_{\theta}(E) E_{\theta}$ accruing to the traditional activity. The private returns $r_{\theta}$ to traditional effort thus coincide with the marginal social returns $\partial Y / \partial E_{\theta}$. On the other hand, unless $\beta_{\theta}=\beta_{\varphi}=0$, the private returns $r_{\varphi}$ to rentseeking effort strictly exceed the marginal social returns $\partial Y / \partial E=r_{\varphi}-\beta_{\theta} Y_{\theta} / E-\beta_{\varphi} r_{\varphi}$.

Rent-seeking Externalities. The divergence between $r_{\varphi}$ and $\partial Y / \partial E$ is why we interpret the $\varphi$-activity as generalized rent-seeking. For example, when $\beta_{\theta}=0$ and in the limiting case $\beta_{\varphi} \rightarrow 1$, there is a fixed "pie" of rents $Y_{\varphi}=k_{\varphi}$ that individuals compete for by exerting effort $e_{\varphi}$. This pie is divided up across agents in proportion to their individual effort $e_{\varphi}$. More generally, when $\beta_{\varphi}<1, Y_{\varphi}$ is increasing but concave in $E$, and a proportion $1-\beta_{\varphi}$ of private rent-seeking earnings are attributable to increasing the size of the rentseeking pie while the remaining portion $\beta_{\varphi}$ is attributable to "skimming" from the portion of the pie that would otherwise have gone to other rent-seekers. Finally, when $\beta_{\theta}>$ 0 , rent-seeking effort additionally reduces the income $Y_{\theta}$ in the traditional activity. The dependence of the returns $r_{\theta}(E)$ and $r_{\varphi}(E)$ on aggregate rent-seeking effort reflects these rent-seeking externalities.

The magnitude of the rent-seeking externality is naturally measured by the Pigouvian correction $t_{\text {Pigou }}$, which aligns the private returns $r_{\varphi}$ to an additional unit of rent-seeking effort $e_{\varphi}$ with the social returns $\partial Y / \partial E$ to this unit. That is, $t_{\text {Pigou }}$ is defined such that

$$
\left(1-t_{\text {Pigou }}\right) r_{\varphi}=\frac{\partial Y}{\partial E} \Rightarrow t_{\text {Pigou }}=\beta_{\varphi}+\frac{1-s}{s} \beta_{\theta}
$$

where $s \equiv Y_{\varphi} / Y$ is the aggregate income share of rent-seeking.

Tax Instruments. There is a benevolent social planner who is aware of the structure of the economy, but who is unable distinguish rent-seeking earnings from traditional earnings. If the planner could separately identify and tax the earnings from rent-seeking efforts, it would optimally impose the tax $t_{\text {Pigou }}$ on rent-seeking earnings. Due to imperfect targeting, however, its only policy tool is a tax on total income, with marginal rate $t$ and lump sum transfer $T$. Faced with such a tax, each agent solves

$$
\max _{e_{\theta}, e_{\varphi}} u\left((1-t)\left(r_{\theta}(E) e_{\theta}+r_{\varphi}(E) e_{\varphi}\right)+T, m\left(e_{\theta}, e_{\varphi}\right)\right),
$$

taking $E$ and hence the returns to each activity as given. It will be useful to rewrite the agent's problem equivalently as 


$$
\max _{l}\left\{\max _{x} u\left((1-t)\left(r_{\theta}(E) \frac{x l}{m(x, 1)}+r_{\varphi}(E) \frac{l}{m(x, 1)}\right)+T, l\right)\right\},
$$

where $x \equiv e_{\theta} / e_{\varphi}$ and we used the fact that $m$ is homogeneous of degree 1 . One can think of this as decomposing the agent's problem into an inner, extensive margin problem of choosing the effort ratio $x$ across activities, and an outer, intensive margin problem of choosing overall effort $l$ (again taking $E$ as given). In particular, the inner maximization is equivalent to choosing the effort ratio $x$ to solve

$$
w_{E} \equiv \max _{x} \frac{r_{\theta}(E) x+r_{\varphi}(E)}{m(x, 1)} .
$$

Then the outer maximum in (3) is

$$
\max _{l} u\left((1-t) w_{E} l+T, l\right) .
$$

Maximization problem (6) indicates that $w_{E}$ is interpretable as a wage. Except for the fact that this wage is endogenous to the aggregate rent-seeking effort in the economy, (6) is a standard problem for an agent facing a linear tax and choosing overall effort $l$.

\subsection{Equilibrium}

An equilibrium is a tuple $\left(t, T, E, E_{\theta}\right)$ with $E=e_{\varphi}^{*}(t, T, E)$ and $E_{\theta}=e_{\theta}^{*}(t, T, E)$, and

$$
T=t\left(r_{\theta}(E) E_{\theta}+r_{\varphi}(E) E\right)
$$

so that the planner's budget is balanced.

Equilibrium Set. The equilibrium condition $E=e_{\varphi}^{*}(t, T, E)$ can be thought of as a fixed point problem for $E$, given any tax system $(t, T)$ : Individuals take the returns and hence $E$ as given when choosing their optimal rent-seeking effort $e_{\varphi}$. In equilibrium, this effort choice has to be consistent with the $E$ that was taken as given. As the following lemma demonstrates, this fixed point problem is well-behaved: it has a unique solution for any $(t, T)$. Indeed, the lemma demonstrates that $E, E_{\theta}, 1-t, l=m\left(E_{\theta}, E\right)$, and $Y$ are all co-monotonic within the set of equilibria.

Lemma 1. If $\left(t, T, E, E_{\theta}\right)$ and $\left(t^{\prime}, T^{\prime}, E^{\prime}, E_{\theta}^{\prime}\right)$ are equilibria, then $1-t \geq 1-t^{\prime} \Leftrightarrow E \geq E^{\prime} \Leftrightarrow$ $E_{\theta} \Leftrightarrow E_{\theta}^{\prime} \Leftrightarrow m\left(E_{\theta}, E\right) \geq m\left(E_{\theta}^{\prime}, E^{\prime}\right) \Leftrightarrow Y\left(E_{\theta}, E\right) \geq Y\left(E_{\theta}^{\prime}, E^{\prime}\right)$.

The proof of Lemma 1, which is in Appendix A, makes use of our regularity assumption $\Delta \sigma>-1+\beta_{\theta}$. Specifically, it shows that $E_{\theta}$ is proportional to $E^{1+\Delta \sigma}$ along the 
equilibrium set, and hence traditional-sector output $Y_{\theta}$ is proportional to $E^{1+\Delta \sigma-\beta_{\theta}}$. If the regularity condition is violated, it is possible that $E_{\theta} \rightarrow 0$ and hence $l=m\left(E_{\theta}, E\right) \rightarrow 0$ as $E \rightarrow 0$. But $Y_{\theta} \rightarrow \infty$ as $E \rightarrow 0$. In other words, it would be feasible to have arbitrarily large output, and hence consumption, with arbitrarily small aggregated effort, because the returns to traditional work explode as aggregate rent-seeking effort vanishes. We focus on the well-behaved case where this is excluded, and equilibria feature the co-monotonicity property established in Lemma 1.

\subsection{Optimal Tax Policy}

By Lemma 1 , the set of equilibria can be parameterized by $E, t, l$, or $Y$. The social planner's problem is to select the equilibrium with the maximum $u(Y, l)$. Per the following proposition, this maximum exists and involves a tax rate $t$ that satisfies a simple and useful condition.

Proposition 1. The optimal tax exists, and satisfies

$$
t=\frac{s_{\text {Pigou }}}{1+(1-s) \sigma \Delta} \geq 0
$$

Intuition. At first glance, one may think that the optimal tax should just equal the share of rent-seeking income $s$ in the economy, multiplied by the Pigouvian tax on rent-seeking $t_{\text {Pigou }}$, i.e. the numerator of (8). Indeed, this would be the optimal correction in partial equilibrium, for an individual agent holding fixed the behavior of all other agents. ${ }^{6}$

The proposition shows, however, that the optimal tax rate diverges from this naive Pigouvian benchmark by an adjustment factor $1 /(1+(1-s) \sigma \Delta)$, which only disappears in the knife-edge cases where the relative returns are fixed (so $\Delta=0$ ) or individuals' effort ratio $e_{\theta} / e_{\varphi}$ is fixed (so $\sigma \rightarrow 0$ ). The intuition is based on the general-equilibrium effects of the tax. The behavior of all other agents will not, in fact, stay fixed as an individual agent adjusts overall effort $l$ in response to a tax change. An increase in one agent's $l$ is associated with an increase in rent-seeking effort $e_{\varphi}$, and hence aggregate $E$, and therefore a corresponding change in the relative returns $r_{\theta}(E) / r_{\varphi}(E)$ (unless $\Delta=0$ ). This relative return change will lead all agents to adjust their effort ratio across the two activities (unless $\sigma \rightarrow 0$ ). The magnitude of this shift depends on the elasticity of substitution $\sigma$ between effort in the two sectors and the elasticity $\Delta$ of relative returns.

\footnotetext{
${ }^{6}$ To see this, note that the private return to overall effort $l$ is $w_{E}$. Since $e_{\varphi}=l / m(x, 1)$ and $e_{\theta}=x l / m(x, 1)$, the social return to overall effort-holding the behavior of the other agents fixed-is $\frac{\partial Y}{\partial E_{\theta}} \frac{x}{m(x, 1)}+\frac{\partial Y}{\partial E_{\varphi}} \frac{1}{m(x, 1)}$. A simple calculation shows that $1-s t_{\text {Pigou }}$ indeed fills the wedge between these two returns.
} 
For example, suppose $\Delta>0$, so an increase in aggregate rent-seeking effort has a stronger negative effect on rent-seeking returns than on traditional returns. Then a tax increase, by lowering individuals' overall and hence rent-seeking effort, increases the relative returns to rent-seeking, and therefore induces a somewhat perverse shift of effort towards rent-seeking. As a result, and per (8), the optimal tax is below the Pigouvian benchmark. On the other hand, when $\Delta<0$, the general equilibrium effects are flipped: the tax, by discouraging effort, induces a reduction in the relative returns and hence a further flow out of rent-seeking. As a result, it is optimal to over-correct compared to the Pigouvian tax.

Relation to Diamond (1973). The formula in (8) bears some resemblance to the results in Diamond (1973). In his model, heterogeneous households demand an externalityproducing consumption good. He shows that the optimal linear tax, when it cannot be differentiated across households, can be expressed as the product of a Pigouvian correction that captures the direct effect of the tax on the demand for the good, and an adjustment term that reflects indirect effects of the changes in consumption across households induced by the direct effect. The adjustment depends in a complicated way on the covariances between the degree to which different households contribute to the externality per unit demanded, the sensitivities of their demands with respect to the externality, and their price sensitivities. In particular, it vanishes when households are identical.

In contrast, our general equilibrium effects result from effort choice along two intensive margins corresponding to two income-earning activities. The divergence we find arises because the tax cannot separately target them, even with identical households. Moreover, we are able to characterize in which direction and by how much the optimal correction should deviate from the Pigouvian tax rate as a function of the product of two simple elasticities. As we demonstrate in the next sections, our results also generalize to a Mirrleesian setting with non-linear income taxes, which allows us to show how the corrective motives for taxation isolated here interact with redistributive motives.

\subsection{An Example}

Putting Numbers. The simplicity of the optimality condition in Proposition 1 facilitates back-of-the-envelope calculations of the adjustment factor. In particular, substitute (2) in (8) to get

$$
t=\frac{s \beta_{\varphi}+(1-s) \beta_{\theta}}{1+(1-s) \Delta \sigma}
$$


so the numerator is just the income-share weighted average of the return elasticities. For example, consider again the case with a fixed "pie" of rents but no cross-activity externalities, so $\beta_{\theta}=0$ and $\beta_{\varphi} \rightarrow 1$. Then $t_{\text {Pigou }} \rightarrow 100 \%, \Delta=1$ and $t=s /(1+(1-s) \sigma)$. If the share of rent-seeking income is, say, $s=10 \%$, the naive Pigouvian benchmark for the tax is also $10 \%$, but the optimal tax is $t=1 /(10+9 \sigma)$. Hence, with $\sigma=1$, the optimal tax is $t \approx 5 \%$, roughly only half as much as the Pigouvian correction; it is even lower with higher substitution elasticities.

Of course, this reasoning ignores the fact that the share of rent-seeking income $s$ at the optimum is endogenous to the tax rate, so we cannot simply treat it as a parameter. However, it is straightforward to compute it for simple parametrizations of the utility function, as any given $t$ implies a unique $s$ in equilibrium. For example, suppose that utility is quasilinear in consumption and isoelastic with effort elasticity $\varepsilon$, so

$$
u(c, l)=c-\frac{l^{1+\frac{1}{\varepsilon}}}{1+\frac{1}{\varepsilon}} .
$$

Appendix A shows that, in equilibrium,

$$
1-t=K s^{\frac{\varepsilon-\sigma}{\varepsilon(1+\sigma)}}\left(\frac{1-s}{S}\right)^{\frac{1+\varepsilon \beta \varphi}{\Delta \varepsilon(1+\sigma)}}
$$

where $K>0$ is some constant that depends on $k_{\theta}$ and $k_{\varphi}$ but is independent of $\sigma$. Equations (9) and (10) thus jointly characterize the optimal $t$ and $s$, for any given parameters. ${ }^{7}$

An Example with Extreme Divergence. More interestingly, we can use this example to formalize the observation that the activity shift effect, and hence the divergence between the optimal tax and the Pigouvian benchmark, can become extreme as $\sigma \rightarrow \infty$, i.e. when individuals always specialize in the sector that delivers the higher return. By our regularity condition, this exercise requires focusing on the case with $\Delta>0$. The following corollary shows that the optimal tax approaches zero even though the Pigouvian benchmark, as well as the share of rent-seeking income, remain strictly positive.

Corollary 1. Suppose $\Delta>0$ and $K<1-\beta_{\varphi}$. As $\sigma \rightarrow \infty$, the unique optimum involves

$$
t \rightarrow 0, \quad s \rightarrow K^{\varepsilon}>0, \quad \text { and } \quad t_{\text {Pigou }} \rightarrow \beta_{\varphi}+\frac{1-K^{\varepsilon}}{K^{\varepsilon}} \beta_{\theta}>0 .
$$

\footnotetext{
${ }^{7}$ Specifically, $K \equiv\left(k_{\varphi} / k_{\theta}\right)^{\left(1+\varepsilon \beta_{\varphi}\right) /(\Delta \varepsilon)} k_{\varphi}^{-1}$. Hence, for any given $t$ and $s$ that satisfy (9), we can, conversely, always reverse-engineer the remaining free parameters, such as the constants $k_{\theta}$ and $k_{\varphi}$, to make sure the equilibrium condition (10) is satisfied, validating the above computations, which treated $s$ as a parameter.
} 
As an illustrative example, consider again the case without cross-activity externalities, with $\beta_{\theta}=0$ and $\beta_{\varphi}=1 / 2$. Then $t_{\text {Pigou }}=50 \%$. Moreover, suppose $\varepsilon=1$ and we choose the free parameter $K$ to approach $1 / 2$ from below. Then the corollary implies that the share of rent-seeking income $s$ also approaches $50 \%$, so the Pigouvian benchmark $s t_{\text {Pigou }} \rightarrow 25 \%$. In other words, half the income in the economy comes from a rent-seeking activity where private returns are twice as high as social marginal returns. Nonetheless, the optimal tax rate approaches 0 , because of the shift effects emphasized here.

To see why, consider a single individual $i$ and suppose she decreases her effort $l$ by a small amount $\delta l$ in response to a tax increase. Since this decreases her rent-seeking effort, the direct effect will be to reduce the externalities she imposes on other agents. But there are also indirect effects: her decrease in rent-seeking effort will increase the relative returns to rent-seeking, leading other individuals to reallocate their efforts towards rentseeking. In fact, with $\sigma \rightarrow \infty$, they re-allocate until the original relative returns, and hence $E$, are restored. (This is because, as $\sigma \rightarrow \infty$, the relative returns are effectively fixed at 1 in any interior equilibrium, to make individuals just indifferent between the two activities.) The net effect is that the total income earned in the economy goes down by exactly $i$ 's reduced earnings $w \delta l$, and, since $E$ is unchanged, the entire change in earnings comes from the traditional sector. To put it another way: the individual's rent-seeking effort is directly unproductive, but, by effectively discouraging other individuals from pursuing additional rent-seeking, it is indirectly productive. With $\sigma \rightarrow \infty$ the indirect productivity exactly equals the private returns to rent-seeking, and the optimal correction, taking general equilibrium effects into account, is exactly zero.

\subsection{Introducing Heterogeneity}

In this section, we have derived our main result, characterizing the divergence between the optimal and Pigouvian correction, in a setting without heterogeneity. In the remainder of the paper, we will show how this result extends to a richer setting where individuals differ in their skills for both activities and the planner has access to a nonlinear income tax and maximizes some social welfare function. ${ }^{8}$ We will see that the intuition emphasized so far will continue to play a prominent role, but will be complemented by the following additional insights:

1. Most obviously, there will be redistributive motives across endogenously heterogeneous wages. We will show how they interact with the purely corrective motives

\footnotetext{
${ }^{8}$ Such a more general framework will also be more suitable for calibration, allowing for a less ad hoc quantification of the optimal divergence (see Section 5).
} 
considered so far.

2. The activity shift effect featured in the denominator of (8) will reappear (and will be labeled effect $S$ below), but it will play a somewhat different role with heterogeneity. Changes in rent-seeking effort at any given wage level affect aggregate returns and will therefore induce activity shifts elsewhere in the wage distribution. As a result, the shift effect $S$ will be global in nature. This will imply an even stronger divergence result, where the optimal correction diverges from the Pigouvian benchmark even at wage levels where the share of rent-seeking income is 1 , and where the (marginal) income tax might appear to be a perfectly targeted instrument. ${ }^{9}$

3. Because the wage distribution is endogenous to aggregate rent-seeking effort, the optimal tax policy will seek to manipulate it in order to relax incentive constraints (labeled effect $I$ below). This Stiglitz (1982)-type emerges more generally in settings with general equilibrium effects (e.g. Rothschild and Scheuer, 2013).

4. More interestingly, there will be heterogeneity not just across wages, but also within wages. For example, there will be individuals who earn the same wage but through a different mix of effort across the two activities, and who will therefore be differentially affected by changes in relative returns induced by changes in taxes. This leads to additional activity shifts (labeled effect $C$ below) as well as redistributive effects (effect $R$ ) within a given wage.

We will provide conditions under which the additional effects $I, C$ and $R$ all reinforce the fundamental shift effect $S$ that we already illustrated in this section, so that the result in Proposition 1 goes through qualitatively.

\section{The General Model}

\subsection{Setup}

Skill Heterogeneity. As before, individuals can pursue two activities: Traditional work and rent-seeking. Individuals are now endowed with a two-dimensional skill vector $(\theta, \varphi) \in \Theta \times \Phi, \Theta=[\underline{\theta}, \bar{\theta}], \Phi=[\underline{\varphi}, \bar{\varphi}]$, where $\theta$ captures an individual's skill for traditional work, and $\varphi$ captures her skill for rent-seeking. In the preceding section, all agents

\footnotetext{
${ }^{9}$ In the online appendix, we provide a simple, stark example with two types that illustrates this point.
} 
had identical skills (normalized to $\theta=\varphi=1$ ). Now, we let skills be distributed with some general, continuous cdf $F: \Theta \times \Phi \rightarrow[0,1]$ and associated continuous pdf $f(\theta, \varphi) .{ }^{10}$

Technology. Let the activity-specific efforts of an individual of type $(\theta, \varphi)$ be denoted by $e_{\theta}(\theta, \varphi)$ and $e_{\varphi}(\theta, \varphi)$, respectively. As before, aggregate output is given by (1), where we now define

$$
E_{\theta} \equiv \int_{\Theta \times \Phi} \theta e_{\theta}(\theta, \varphi) d F(\theta, \varphi) \text { and } E \equiv \int_{\Theta \times \Phi} \varphi e_{\varphi}(\theta, \varphi) d F(\theta, \varphi)
$$

as the aggregate effective (i.e., skill-weighted) efforts in the traditional and rent-seeking activities, respectively. Correspondingly, individuals earn income in proportion to their effective effort in each activity, so $y(\theta, \varphi)=r_{\theta}(E) \theta e_{\theta}(\theta, \varphi)+r_{\varphi}(E) \varphi e_{\varphi}(\theta, \varphi)$. To capture the distinction between rent-seeking and traditional work, we again assume that both returns $r_{\theta}(E)$ and $r_{\varphi}(E)$ are decreasing in aggregate rent-seeking effort $E$, but are independent of traditional effort $E_{\theta}$. As a result, the private return to effective effort coincides with the social marginal product in the traditional activity, but exceeds it in the rent-seeking activity, as in the previous section. Let the elasticities of the returns with respect to $E$ be denoted by $\beta_{\theta}(E)=-r_{\theta}^{\prime}(E) E / r_{\theta}(E)$ and $\beta_{\varphi}(E)=-r_{\varphi}^{\prime}(E) E / r_{\varphi}(E)$ and the elasticity of the relative return to traditional work $r_{\theta} / r_{\varphi}$ by $\Delta(E) \equiv \beta_{\varphi}(E)-\beta_{\theta}(E) . \Delta(E)$ measures the relative importance of within- versus across-activity externalities.

It is worth pointing out that this specification of technology is very general. In particular, it is implied by any model where (i) each unit of effective effort in a given activity has the same private return, (ii) effort in the $\Theta$-activity imposes no externalities, and (iii) effort in the $\Phi$-activity imposes at least weakly negative externalities on both activities. We also emphasize that this technology does not require firms or employers. We can assume that each worker is self-employed and reaps the return to his effort directly. However, the returns to both traditional and rent-seeking effort are determined in general equilibrium, by the supply of effort of all other workers. Finally, note that property (i) implies that the rent-seeking externality works through individual returns to effective effort. It therefore rules out the uniform absolute reduction in other individuals' incomes due to rent-seeking (considered, for instance, in Piketty et al., 2014, and Lockwood et al., 2015), which is independent of effort. Indeed, the interesting relative return effects on activity choice that we explore in the following arise precisely because we allow rent-seeking to have differential effects on the returns to different types of effort. ${ }^{11}$

\footnotetext{
${ }^{10}$ See Section 6 for a discussion of how our framework can be extended to capture further heterogeneity in the disutility from, or taste for, working in the two activities.

${ }^{11}$ In the online appendix, we discuss several examples and applications that can be captured by our
} 
Preferences. We consider the same utility function as in Section 2, given by $U\left(c, e_{\theta}, e_{\varphi}\right)=$ $u\left(c, m\left(e_{\theta}, e_{\varphi}\right)\right) \equiv u(c, l)$. In the appendix, we will show that all our results hold for a general effort aggregator $m\left(e_{\theta}, e_{\varphi}\right)$ that is increasing in both arguments, continuously differentiable, quasiconvex and linear homogeneous. ${ }^{12}$ To simplify the exposition, in the main text we will focus our discussion on the special case where individuals always specialize in one activity. This always obtains when $m\left(e_{\theta}, e_{\varphi}\right)$ is linear, and corresponds most naturally to the interpretation of activities as tied to sectors or occupations (which is also the application we will consider in our numerical illustration in Section 5). In this case, one can think of individuals as making an extensive margin choice, picking one of the two sectors depending on relative returns, and an intensive margin choice, picking the amount of effort they want to provide in the chosen sector, as in the Roy model considered in Rothschild and Scheuer (2013).

When $m$ is strictly quasiconvex, individuals generally choose some interior effort mix $x=e_{\theta} / e_{\varphi}$. Still, as seen in the previous section, one can decompose the individual's problem into two very similar subproblems: a choice of the effort ratio $x$, which (due to the linear homogeneity of $m$ ) is again pinned down by the relative returns to the two activities, and an intensive margin choice of $l$. This can capture situations where an individual's job involves a mixture of both rent-seeking and traditional, productive activities.

In the Ramsey model from the previous section, we saw that as we approached the case with linear $m$, and hence the substitution elasticity $\sigma \rightarrow \infty$, we could obtain unbounded shift effects and therefore extreme results for optimal taxes. With heterogeneity, however, this connection no longer holds, because a linear $m$ at the individual level does not imply an infinite substitution elasticity at the aggregate level. Intuitively, we will see that most individuals' sectoral choices are not responsive at all to small changes in relative returns (as their skills are such that they strictly prefer working in one of the two sectors). Only the small set of individuals who were close to indifferent between the two sectors before the change do respond, leading to a finite and well-behaved aggregate shift effect even in a model with full specialization.

framework, including the contests and races with winner-takes-all compensation that are widespread in finance, law, and research.

${ }^{12}$ Note that, since $u$ is left general, this allows for preferences $\tilde{u}\left(c, \tilde{m}\left(e_{\theta}, e_{\varphi}\right)\right)$ where $\tilde{m}$ is homothetic but not linear homogeneous: then there exist transformations $u$ and $m$ of $\tilde{u}$ and $\tilde{m}$ such that $\tilde{u}\left(c, \tilde{m}\left(e_{\theta}, e_{\varphi}\right)\right)=$ $u\left(c, m\left(e_{\theta}, e_{\varphi}\right)\right)$ for all $\left(c, e_{\theta}, e_{\varphi}\right)$ with linear homogeneous $m$. An example is $\hat{u}(c)-h_{\theta}\left(e_{\theta}\right)-h_{\varphi}\left(e_{\varphi}\right)$ with $h_{\theta}($. and $h_{\varphi}($.$) homogeneous of the same degree.$ 


\subsection{Implementation}

A Direct Mechanism. We respectively denote the consumption, effort, utility and activity assigned to an individual of type $(\theta, \varphi)$ by $c(\theta, \varphi), l(\theta, \varphi), V(\theta, \varphi) \equiv u(c(\theta, \varphi), l(\theta, \varphi))$ and $S(\theta, \varphi) \in\{\Theta, \Phi\}$. We also define an individual's wage as the return to effort in the assigned activity, so that

$$
w_{E}(\theta, \varphi)=\left\{\begin{array}{lll}
r_{\theta}(E) \theta & \text { if } & S(\theta, \varphi)=\Theta \\
r_{\varphi}(E) \varphi & \text { if } & S(\theta, \varphi)=\Phi
\end{array}\right.
$$

and we can write an individual's income simply as $y(\theta, \varphi)=w_{E}(\theta, \varphi) l(\theta, \varphi)$. As is standard, we assume the single crossing property, i.e., that the marginal rate of substitution between $y$ and $c,-u_{l}(c, y / w) /\left(w u_{c}(c, y / w)\right)$, is decreasing in $w$.

We now describe a direct mechanism where individuals announce their privately known type $(\theta, \varphi)$ and then get allocated $c(\theta, \varphi), y(\theta, \varphi)$, and $S(\theta, \varphi)$. We will then link this to the implementation through a nonlinear income tax schedule $T(y)$ using the results in Rothschild and Scheuer (2013). In line with the imperfect targeting considered in the Section 2, we take only income and consumption as de facto contractible for the government, but not an individual's skill type, sector, wage and effort. ${ }^{13}$ The resulting incentive constraints that guarantee truth-telling of $(\theta, \varphi)$ in the direct mechanism are:

$$
u\left(c(\theta, \varphi), \frac{y(\theta, \varphi)}{w_{E}(\theta, \varphi)}\right) \geq \max \left\{u\left(c\left(\theta^{\prime}, \varphi^{\prime}\right), \frac{y\left(\theta^{\prime}, \varphi^{\prime}\right)}{r_{\theta}(E) \theta}\right), u\left(c\left(\theta^{\prime}, \varphi^{\prime}\right), \frac{y\left(\theta^{\prime}, \varphi^{\prime}\right)}{r_{\varphi}(E) \varphi}\right)\right\} \forall\left(\theta^{\prime}, \varphi^{\prime}\right)
$$

since type $(\theta, \varphi)$ can imitate any other type $\left(\theta^{\prime}, \varphi^{\prime}\right)$ by earning $\left(\theta^{\prime}, \varphi^{\prime}\right)^{\prime}$ s income either in the $\Theta$ - or the $\Phi$-activity.

An Income Tax Implementation. The following lemma, due to Rothschild and Scheuer (2013), shows that any incentive compatible allocation can be implemented by offering a nonlinear income tax $T(y)$.

Lemma 2. Any incentive compatible allocation $\{c(\theta, \varphi), y(\theta, \varphi), S(\theta, \varphi), E\}$ is such that

$$
w_{E}(\theta, \varphi)=\max \left\{r_{\theta}(E) \theta, r_{\varphi}(E) \varphi\right\} \text { and } S(\theta, \varphi)=\left\{\begin{array}{ccc}
\Theta & \text { if } & r_{\theta}(E) \theta>r_{\varphi}(E) \varphi \\
\Phi & \text { if } & r_{\theta}(E) \theta<r_{\varphi}(E) \varphi
\end{array}\right.
$$

(ii) $u(c(\theta, \varphi), y(\theta, \varphi) / w)=u\left(c\left(\theta^{\prime}, \varphi^{\prime}\right), y\left(\theta^{\prime}, \varphi^{\prime}\right) / w\right)$ for all $(\theta, \varphi),\left(\theta^{\prime}, \varphi^{\prime}\right)$ such that $w_{E}(\theta, \varphi)=$ $w_{E}\left(\theta^{\prime}, \varphi^{\prime}\right)=w$;

\footnotetext{
${ }^{13}$ See Section 6 for a discussion of these assumptions.
} 
(iii) it can be implemented by offering a nonlinear income tax schedule $T(y)$ and letting agents choose their preferred $(c, y)$-bundle from the resulting budget set $B=\{(c, y) \mid c \leq y-T(y)\}$.

The first two properties say that individuals always specialize in the activity where their return to effort is higher and that individuals with the same wage must obtain the same utility. The third property establishes that the principle of taxation holds. The second property does not rule out that two individuals with the same wage (but who specialize in different activities) choose different $(c, y)$-bundles. Nonetheless, as argued in Rothschild and Scheuer (2013), we can restrict attention to allocations $\{c(w), y(w), E\}$ that pool all same-wage individuals at the same $(c, y)$-bundle. The reason is that such pooling can always be done in an incentive compatible and resource feasible way, and such that it does not affect $E$ or, by property (ii), utilities.

In Appendix B, we show how these results generalize to the case where individuals do not necessarily specialize in one of the two activities, but pursue both simultaneously. Instead of assigning an activity from the binary set $\{\Theta, \Phi\}$, an allocation then specifies the share of income $q_{E} \in[0,1]$ that an individual earns in the traditional activity (and conversely $1-q_{E}$ is earned through rent-seeking). Defining the wage analogously to (5) in Section 2, our analysis goes through and Lemma 2 applies. In particular, any individual's wage $w_{E}$ and income share $q_{E}$ are fully pinned down by aggregate rent-seeking effort $E$ but are independent of the rest of the allocation. Because all individuals with the same wage have the same preferences over $(c, y)$-bundles given by $u(c, y / w)$, the screening problem can again be reduced to offering allocations $\{c(w), y(w)\}$ that only condition on wages, which in turn are endogenous to $E$.

\section{Optimal Non-linear Income Taxation}

In this section, we characterize the set of Pareto efficient nonlinear income tax schedules. This allows us to compare the optimal and Pigouvian corrections in this richer setting.

\subsection{Pareto Optima}

Wage Distributions. Lemma 2 showed that fixing $E$ determines the wage $w_{E}(\theta, \varphi)$ and activity choice of each type $(\theta, \varphi)$. Together with the two-dimensional skill distribution $F(\theta, \varphi)$, it therefore determines a one-dimensional wage distribution with cdf

$$
F_{E}(w)=F\left(\frac{w}{r_{\theta}(E)}, \frac{w}{r_{\varphi}(E)}\right)
$$


and sectoral densities

$$
f_{E}^{\theta}(w)=\frac{1}{r_{\theta}(E)} \int_{\underline{\varphi}}^{w / r_{\varphi}(E)} f\left(\frac{w}{r_{\theta}(E)}, \varphi\right) d \varphi, \quad f_{E}^{\varphi}(w)=\frac{1}{r_{\varphi}(E)} \int_{\underline{\theta}}^{w / r_{\theta}(E)} f\left(\theta, \frac{w}{r_{\varphi}(E)}\right) d \theta
$$

with corresponding $\operatorname{cdfs} F_{E}^{\theta}(w)$ and $F_{E}^{\varphi}(w)$ and with $f_{E}(w)=f_{E}^{\theta}(w)+f_{E}^{\varphi}(w)$. Hence, $f_{E}^{\varphi}(w) / f_{E}(w)$ is the share of rent-seekers at wage level $w$. We denote the support of the wage distribution for any $E$ by $\left[\underline{w}_{E}, \bar{w}_{E}\right]$, where $\underline{w}_{E}=w_{E}(\underline{\theta}, \underline{\varphi})$ and $\bar{w}_{E}=w_{E}(\bar{\theta}, \bar{\varphi}) .{ }^{14}$

Pareto Weights. We use general cumulative Pareto weights $\Psi(\theta, \varphi)$ in $(\theta, \varphi)$-space with the corresponding density $\psi(\theta, \varphi)$ to obtain Pareto efficient allocations. The social planner maximizes $\int_{\Theta \times \Phi} V(\theta, \varphi) d \Psi(\theta, \varphi)$ subject to resource and self-selection constraints. Completely analogously to the wage distributions above, for any given $E$, we can derive Pareto weights over wages $\Psi_{E}(w)$, as well as their density and decomposition across activities $\psi_{E}(w)=\psi_{E}^{\theta}(w)+\psi_{E}^{\varphi}(w)$, from $\Psi(\theta, \varphi)$. We are particularly interested in the regular case in which the planner assigns greater weight to low-wage individuals, i.e., where $\psi_{E}(w) / f_{E}(w)$ is non-increasing in $w$ for any $E .{ }^{15}$

Elasticities. Any incentive compatible allocation $\{c(w), y(w), E\}$ implies total effort and utility $l(w) \equiv y(w) / w$ and $V(w) \equiv u(c(w), l(w))$. We denote the resulting uncompensated and compensated wage elasticities of total effort $l$ by $\varepsilon^{u}(w)$ and $\varepsilon^{c}(w)$, respectively.

A Decomposition of the Pareto Problem. As in Rothschild and Scheuer (2013), we can decompose the problem of finding Pareto optimal allocations into two steps. The first step involves finding the optimal level of aggregate rent-seeking effort $E$. We call this the "outer" problem. The second (which we call the "inner" problem) involves finding the optimal resource-feasible and incentive-compatible allocation for a given level of $E$. This inner problem is an almost standard Mirrlees problem; the only difference is that the induced level of aggregate effective rent-seeking effort has to be consistent with the level of $E$ that we are fixing for the inner problem. For some given Pareto weights $\Psi(\theta, \varphi)$ (and hence induced weights $\Psi_{E}(w)$ ), we therefore define the inner problem as follows (where

\footnotetext{
${ }^{14}$ We show in Appendix $C$ how these definitions can be extended to the case of a general effort aggregator $m$, with the interpretation that $f_{E}^{\theta}(w)$ and $f_{E}^{\varphi}(w)$ are the average value of $q$ (respectively $1-q$ ) at wage level $w$. Hence, more generally, $f_{E}^{\varphi}(w) / f_{E}(w)$ is the share of rent-seeking income at $w$.

${ }^{15}$ For example, consider the case of relative Pareto weights where $\Psi(\theta, \varphi)=\tilde{\Psi}(F(\theta, \varphi))$ for some increasing function $\tilde{\Psi}:[0,1] \rightarrow[0,1]$. Then these Pareto weights are regular whenever $\tilde{\Psi}$ is weakly concave.
} 
$c(V, e)$ is the inverse function of $u(c, l)$ with respect to $c)$ :

$$
W(E) \equiv \max _{V(w), l(w)} \int_{\underline{w}_{E}}^{\bar{w}_{E}} V(w) d \Psi_{E}(w)
$$

subject to

$$
\begin{gathered}
V^{\prime}(w)+u_{l}(c(V(w), l(w)), l(w)) \frac{l(w)}{w}=0 \quad \forall w \in\left[\underline{w}_{E}, \bar{w}_{E}\right] \\
r_{\varphi}(E) E-\int_{\underline{w}_{E}}^{\bar{w}_{E}} w l(w) f_{E}^{\varphi}(w) d w=0 \\
\int_{\underline{w}_{E}}^{\bar{w}_{E}} w l(w) f_{E}(w) d w-\int_{\underline{w}_{E}}^{\bar{w}_{E}} c(V(w), l(w)) f_{E}(w) d w \geq 0 .
\end{gathered}
$$

We employ the standard Mirrleesian approach of optimizing directly over allocations, i.e., over effort $l(w)$ and consumption or, equivalently, utility $V(w)$ profiles. The social planner maximizes a weighted average of individual utilities $V(w)$ subject to three constraints. (15) is a standard resource constraint and (14) ensures that aggregate effective effort in the rent-seeking activity indeed sums up to $E$ (or, equivalently, rent-seeking incomes sum to $\left.r_{\varphi}(E) E\right)$. Finally, the allocation $V(w), l(w)$ needs to be incentive compatible, i.e.,

$$
V(w) \equiv u(c(w), l(w))=\max _{w^{\prime}} u\left(c\left(w^{\prime}\right), \frac{l\left(w^{\prime}\right) w^{\prime}}{w}\right) .
$$

It is a well-known result that under single-crossing, the global incentive constraints (16) are equivalent to the local incentive constraints (13) and the monotonicity constraint that income $y(w)$ must be non-decreasing in $w .{ }^{16}$ We follow the standard approach of dropping the monotonicity constraint, which can easily be checked ex post (as we do for the numerical simulations in Section 5). If the solution to problem (12) to (15) does not satisfy it, optimal bunching would need to be considered. Accounting for bunching is conceptually straightforward and does not substantively effect our analysis, so, for simplicity, we abstract from bunching henceforth.

Once a solution $V(w), l(w)$ to the inner problem has been found, the resulting welfare is given by $W(E)$. The outer problem is then simply $\max _{E} W(E)$. It is straightforward to show that a solution to the inner problem exists for any $E$ (see Rothschild and Scheuer, 2014, for details) and that, at any $E$ for which individuals work in both activities, $W(E)$ is continuous, so that the outer problem has a solution over any compact set of Es. ${ }^{17}$

\footnotetext{
${ }^{16}$ See, for instance, Fudenberg and Tirole (1991), Theorems 7.2 and 7.3.

${ }^{17}$ Compactness would be ensured, for instance, by a standard Inada condition $u_{l}(c, l) \rightarrow-\infty$ as $l \uparrow \bar{l}$ for some $\bar{l}<\infty$.
} 


\subsection{Marginal Tax Rate Formulas from the Inner Problem}

Solving the inner problem (12) to (15) for a given $E$ yields the following optimal marginal tax rate formula:

Proposition 2. The marginal tax rate in any Pareto optimum is such that

$$
\begin{gathered}
1-T^{\prime}(y(w))=\left(1-\xi \frac{f_{E}^{\varphi}(w)}{f_{E}(w)}\right)\left(1+\frac{\eta(w)}{w f_{E}(w)} \frac{1+\varepsilon^{u}(w)}{\varepsilon^{c}(w)}\right)^{-1} \text { with } \\
\eta(w)=\int_{w}^{\bar{w}_{E}}\left(1-\frac{\psi_{E}(x)}{f_{E}(x)} \frac{u_{c}(x)}{\lambda}\right) \exp \left(\int_{w}^{x}\left(1-\frac{\varepsilon^{u}(s)}{\varepsilon^{c}(s)}\right) \frac{d y(s)}{y(s)}\right) f_{E}(x) d x
\end{gathered}
$$

for all $w \in\left[\underline{w}_{E}, \bar{w}_{E}\right]$, where $\lambda$ is the multiplier on the resource constraint (15) and $\lambda \xi$ the multiplier on the consistency constraint (14).

These formulas are the same as those for a standard Mirrlees model (see e.g. equations (15) to (17) in Saez, 2001), with the only difference that, at each wage, marginal keep shares $1-T^{\prime}(y(w))$ are scaled down by the correction factor $1-\xi f_{E}^{\varphi}(w) / f_{E}(w)$, where $\xi$ is the (normalized) Lagrange multiplier on constraint (14) and $f_{E}^{\varphi}(w) / f_{E}(w)$ is the fraction of incomes earned in the rent-seeking activity at wage level $w$. This optimal local correction, which makes agents internalize the rent-seeking externality, is thus proportional to the relative importance of rent-seeking at $w$ and the shadow cost of the consistency constraint (14). As usual, the term $\eta(w)$ captures the redistributive motives of the government and income effects from the terms in the exponential function. A particularly simple formula can be obtained from (17) with quasilinear preferences $u(c, l)=c-h(l)$, where income effects disappear, as in Diamond (1998). Then $u_{c}(w)=\lambda=1$ and $\varepsilon^{u}(w)=\varepsilon^{c}(w) \forall w$, so that $\eta(w)=\Psi_{E}(w)-F_{E}(w)$. Hence $T^{\prime}(y(w)) \geq 0$ at all income levels under regular Pareto weights, and the marginal tax rate is increasing in the degree to which $\Psi_{E}(w)$ shifts weight to low-wage individuals compared to $F_{E}(w)$.

Under any preference assumptions, the top marginal tax rate is given by $T^{\prime}\left(y\left(\bar{w}_{E}\right)\right)=$ $\xi f_{E}^{\varphi}\left(\bar{w}_{E}\right) / f_{E}\left(\bar{w}_{E}\right)$, or simply $\xi$ if all income at the top is from rent-seeking. We next consider the outer problem in order to explore the determination of $E$ and $\xi$.

\subsection{Optimal Rent-Seeking Effort from the Outer Problem}

Our main goal in the following is to compare $\xi$ to the Pigouvian tax $t_{\text {Pigou }}$ defined in (2) in Section 2, i.e., the tax that aligns the private and social returns to rent-seeking effort. ${ }^{18}$

\footnotetext{
${ }^{18}$ We show in the online appendix that $t_{\text {Pigou }}$ can be interpreted, as in Section 2, as the optimal corrective tax if, in addition to levying an optimal nonlinear income tax, the government could directly tax rent-
} 
The key question will be how $\xi$-interpretable the optimal externality correction in our model with imperfect targeting — differs from the targeted instrument benchmark $t_{\text {Pigou }}$.

Welfare Effects of Changing $E$. To be able to answer this question, Lemma 3 provides an important auxiliary result, namely a decomposition of the welfare effect of marginal changes in $E$ that will be useful in the following.

Lemma 3. The welfare effect of a marginal change in aggregate rent-seeking effort $E$ is

$$
W^{\prime}(E)=\lambda r_{\varphi}(E)\left(\xi-t_{\text {Pigou }}\right)+\frac{\Delta(E)}{E}[I+R+\xi \lambda(C+S)]
$$

where

$$
\begin{gathered}
I \equiv \lambda \int_{\underline{w}_{E}}^{\bar{w}_{E}} \eta(w) w \frac{V^{\prime}(w)}{u_{c}(w)} \frac{d}{d w}\left(\frac{f_{E}^{\varphi}(w)}{f_{E}(w)}\right) d w \\
R \equiv \int_{\underline{w}_{E}}^{\bar{w}_{E}} V^{\prime}(w) w \frac{f_{E}^{\theta}(w) f_{E}^{\varphi}(w)}{f_{E}(w)}\left(\frac{\psi_{E}^{\theta}(w)}{f_{E}^{\theta}(w)}-\frac{\psi_{E}^{\varphi}(w)}{f_{E}^{\varphi}(w)}\right) d w \\
C \equiv \int_{\underline{w}_{E}}^{\bar{w}_{E}} w^{2} l^{\prime}(w) \frac{f_{E}^{\theta}(w) f_{E}^{\varphi}(w)}{f_{E}(w)} d w
\end{gathered}
$$

and

$$
S \equiv \int_{\underline{w}_{E}}^{\bar{w}_{E}} w^{2} l(w) f\left(\frac{w}{r_{\theta}(E)}, \frac{w}{r_{\varphi}(E)}\right) d w \geq 0
$$

Direct Effects. The first term $\lambda \xi r_{\varphi}(E)$ in (19) is simply the direct effect of a change in $E$ on the consistency constraint (14), holding effort and sector constant for each individual. Similarly, the second term $-\lambda t_{\text {Pigou }} r_{\varphi}(E)$ captures the effect of changing sectoral returns on the resource constraint (15), holding effort, sector and consumption fixed for all types. ${ }^{19}$ In fact, when $\Delta(E)=0, E$ has no effect on relative returns $r_{\theta}(E) / r_{\varphi}(E)$. So changing $E$ while holding the effort and activity choice for each type $(\theta, \varphi)$ fixed is compatible with the incentive constraints (11). By an envelope argument, then, $W^{\prime}(E)=$ $\lambda\left(\xi-t_{\text {Pigou }}\right) r_{\varphi}(E)$, consistent with (19).

When $\Delta(E) \neq 0$, the change in relative returns drives a wedge between $\xi$ and $t_{\text {Pigou }}$ since holding allocations fixed is no longer incentive compatible, and there are additional welfare effects from a change in $E$ captured by the four effects in (20) to (23), which are parallel to those in Rothschild and Scheuer (2013). In discussing them, we focus on the seeking income (see Proposition 1 there).

${ }^{19}$ To see this, differentiate with respect to $E^{\prime}$ the total income in the economy $r_{\theta}\left(E^{\prime}\right) E_{\theta}+r_{\varphi}\left(E^{\prime}\right) E$ to get (evaluated at $\left.E^{\prime}=E\right) r_{\theta}^{\prime}(E) E_{\theta}+r_{\varphi}^{\prime}(E) E=\partial \Upsilon\left(E_{\theta}, E\right) / \partial E-r_{\varphi}(E)=-r_{\varphi}(E) t_{\text {Pigou }}$. 
case $\Delta(E)>0$, so that an increase in $E$ increases the relative return to traditional work $r_{\theta} / r_{\varphi}$. The opposite case is analogous with reversed signs.

Activity Shift Effect. A change in $E$ causes an activity or sectoral shift, analogous to the one found in the Ramsey model in Section 2. An increase in $E$ (and thus $r_{\theta} / r_{\varphi}$ ) leads some previously indifferent individuals to switch from rent-seeking to traditional work; $S$ measures the effort shifted as a result. ${ }^{20}$ In the representative agent Ramsey model of Section 2, the activity shift was the sole driver of the divergence between the optimal and the Pigouvian correction.

Novel Effects due to Heterogeneity. The additional effects $C, R$ and $I$ arise from the rich heterogeneity in the Mirreleesian framework. Specifically, they arise because a change in $E$ has differential wage effects on distinct individuals.

The term $I$ is a generalized Stiglitz (1982) effect, which arises because the magnitude of the wage effects varies across the wage distribution. If $\Delta>0$ and the share of income earned through rent-seeking is locally increasing in $w$ (i.e., $d\left(f_{E}^{\varphi}(w) / f_{E}(w)\right) / d w>0$ ), then an increase in $E$ leads to a local compression of the wage distribution, as returns in the high-wage activity fall relative to the low-wage activity. This yields a welfareimproving easing of the local incentive constraints (13) if they are binding downwards $(\eta(w) \geq 0)$. I therefore vanishes if there are no redistributive motives (e.g. with quasilinear preferences and $\tilde{\Psi}(F)=F \forall F)$, so that $\eta(w)=0$ for all $w$.

Moreover, a change in $E$ has different effects on the wages of distinct individuals who originally earned the same wage $w$. In particular, the wage of a traditional worker at $w$ falls by $r_{\theta}^{\prime}(E) w / r_{\theta}(E)=\beta_{\theta}(E) w / E$ whereas the wage of a rent-seeker falls by $\beta_{\varphi}(E) w / E$. Hence, when $\Delta(E)>0$, the rent-seekers at $w$ see their wages fall by more than the average for the wage $w$-workers, and the traditional workers see their wages fall by less. The term $C$ arises because, by changing their wages differentially in the face of a fixed effort schedule $l(w)$, an increase in $E$ in effect re-allocates effort across the rent-seekers and traditional workers who are originally pooled. In particular, for an increasing (in $w$ ) effort schedule, a rise in $E$ results in a re-allocation of effort from rent-seekers (who effectively move down along the schedule) to traditional workers (who move up) at any given $w$.

\footnotetext{
${ }^{20}$ We show in Appendix C.3 how $S$ can be generalized to allow for general $m$, in which case it will also incorporate continuous changes in the effort ratio $e_{\theta} / e_{\varphi}$ in response to the relative return change, as in Section 2.
} 
Hence, $C$ reinforces $S$ if $l^{\prime}(w) \geq 0 .{ }^{21}$

The term $R$ arises from the analogous reallocation of utility $V(w)$ from rent-seekers to traditional workers at the same initial wage, which is why it shows up in parallel to the redistributive Stiglitz-term $I$. It disappears with relative welfare weights $\Psi(\theta, \varphi)=$ $\tilde{\Psi}(F(\theta, \varphi))$, since then $\psi_{E}^{\theta}(w) / f_{E}^{\theta}(w)=\psi_{E}^{\varphi}(w) / f_{E}^{\varphi}(w)$ for all $w, E$. Otherwise, it is welfare improving when the planner puts more weight on traditional workers at each wage $\left(\psi_{E}^{\theta}(w) / f_{E}^{\theta}(w)>\psi_{E}^{\varphi}(w) / f_{E}^{\varphi}(w)\right)$ and vice versa. ${ }^{22}$

\subsection{Marginal Tax Rate Results}

Comparing the Optimal and Pigouvian Corrections. We can now use Lemma 3 to derive the following relationship between $\xi$ and $t_{\text {Pigou }}$ at any interior Pareto optimum. Setting $W^{\prime}(E)=0$ yields:

$$
\xi=t_{\text {Pigou }}\left(1-\frac{1}{\lambda t_{\text {Pigou }}} \frac{\Delta(E)}{Y_{\varphi}(E)}(I+R)\right) /\left(1+\frac{\Delta(E)}{Y_{\varphi}(E)}(C+S)\right) .
$$

In a one-activity model with only the rent-seeking activity available and $f_{E}^{\theta}(w)=0$ for all $w$, we mechanically have $I=R=C=S=0$ and therefore $\xi=t_{\text {Pigou }}=\beta_{\varphi}(E)$. The tax formula (17) then implies that the correction factor by which marginal keep shares are scaled down compared to the standard formula is uniform and given by $1-t_{\text {Pigou }}$. This can be understood as a two-step correction as in Kopczuk (2013): first tax all wages by $t_{\text {Pigou }}$ to correct the rent-seeking externality. Then apply the standard optimal tax formula, as in a Mirrlees model without externalities, with the corrected wages $\left(1-t_{\text {Pigou }}\right) w$. In particular, the top marginal tax rate is just $T^{\prime}\left(y\left(\bar{w}_{E}\right)\right)=t_{\text {Pigou }}$.

In the general case where both activities take place, the optimal correction $\xi$ deviates from $t_{\text {Pigou }}$ due to the relative return effects (20) to (23) whenever $\Delta(E) \neq 0$. Based on the discussion in the previous subsection and (24), the following proposition collects conditions that determine this comparison.

${ }^{21}$ In particular, the average wage decline for those originally pooled at $w$ is

$$
\frac{f_{E}^{\theta}(w)}{f_{E}(w)} \beta_{\theta}(E) \frac{w}{E}+\frac{f_{E}^{\varphi}(w)}{f_{E}(w)} \beta_{\varphi}(E) \frac{w}{E}
$$

Hence, the change in effort induced by the rise in $E$, relative to the wage- $w$ average, is $l^{\prime}(w)\left(f_{E}^{\theta}(w) / f_{E}(w)\right) \Delta(E) w / E$ for a rent-seeker with original wage $w$. Multiplying this with $w$ and the share of rent-seekers at $w$, the change in rent-seeking income is thus $w^{2} l^{\prime}(w)\left(f_{E}^{\varphi}(w) f_{E}^{\theta}(w) / f_{E}(w)^{2}\right) \Delta(E) / E$. Summing over all $w$ 's therefore leads to the effect $C$ in (22) on the consistency constraint (14).

${ }^{22}$ In Appendix C.3, we prove Lemma 3 for general $m$ and show that the decomposition (19) goes through, $I$ and $R$ are unaffected and $C$ and $S$ can be generalized in a straightforward way, with the same intuition and properties as discussed here. 
Proposition 3. In any regular Pareto optimum, $\xi>0$. If in addition: (i) effort $l(w)$ is weakly increasing in w; (ii) marginal utility of consumption $u_{c}(c(w), l(w))$ is weakly decreasing in $w$; (iii) the share of rent-seeking incomes $f_{E}^{\varphi}(w) / f_{E}(w)$ is weakly increasing in $w$; and (iv) the welfare weights on traditional workers are not smaller than those on rent-seekers at each w, so that $\psi_{E}^{\theta}(w) / f_{E}^{\theta}(w) \geq \psi_{E}^{\varphi}(w) / f_{E}^{\varphi}(w) \forall w$, then

$$
\xi \lesseqgtr t_{\text {Pigou }} \text { if } \quad \Delta(E) \gtreqless 0 .
$$

Combined with the marginal tax rate formula in Proposition 2, this result has clear implications for Pareto optimal tax schedules. For instance, under the conditions in Proposition 3 and if all income at the top is earned through rent-seeking $\left(f_{E}^{\varphi}\left(\bar{w}_{E}\right) / f_{E}\left(\bar{w}_{E}\right)=1\right)$, then $T^{\prime}\left(y\left(\bar{w}_{E}\right)\right)=\xi \lesseqgtr t_{\text {Pigou }}$ iff $\Delta(E) \gtreqless 0$. Hence, if e.g. $\Delta(E)>0$, the top marginal tax rate is less than the Pigouvian correction $t_{\text {Pigou }}$ even when all top earners are exclusively active in the rent-seeking activity. In this sense, we obtain a stronger divergence result compared to the Ramsey model from Section 2, where the divergence vanished when the share of rent-seekers was $1 .^{23}$ At other income levels, the optimal correction $\xi$ is still less than $t_{\text {Pigou }}$ by Proposition 3 , but of course gets combined with the redistributive components of the marginal tax rate according to (17).

Intuition. The divergence of the optimal correction $\xi$ from $t_{\text {Pigou }}$ directly reflects the fact that the income tax is an imperfect tool for externality correction, even in income brackets where all income comes from rent-seeking. This is because, as the discussion above highlights, the effects of the externality $E$ are non-uniform (whenever $\Delta(E) \neq 0$ ) and global in nature. When $\Delta(E)>0$, taxing rent-seeking intensive portions of the income distribution at a higher rate directly discourages effort at those income levels, lowering $E$ and helping to correct the externality. As in Section 2, however, a lower $E$ raises the relative returns to rent-seeking globally, encouraging a shift into this activity. This implies a smaller-thanPigouvian optimal correction. On the other hand, when $\Delta(E)<0$, higher taxes directly discourage the externality-causing activity, and, since this lowers the relative returns to rent-seeking, indirectly encourage effort-shifting away from rent-seeking. As a result, the optimal $\xi$ exceeds $t_{\text {Pigou }}$ and, for instance, the optimal top marginal tax rate over-corrects compared to the Pigouvian rate.

As discussed in Section 4.3, apart from the shift effect $S \geq 0$, which is the analog of the shift effect found in Section 2, there are three additional relative return effects $C, I$, and $R$ that arise due to heterogeneity. The assumptions in Proposition 3 are sufficient to ensure

\footnotetext{
${ }^{23}$ Of course, a fortiori we obtain $0 \leq T^{\prime}\left(y\left(\bar{w}_{E}\right)\right)=\xi f_{E}^{\varphi}\left(\bar{w}_{E}\right) / f_{E}\left(\bar{w}_{E}\right)<t_{\text {Pigou }}$ if the share of income from rent-seeking is less than one at the top and $\Delta(E)>0$.
} 
that they are also non-negative and thus reinforce $S$. Note, however, that these are only sufficient conditions, so that the comparison between $\xi$ and $t_{\text {Pigou }}$ can hold even when they are violated for some wage levels. For instance, with relative Pareto weights and quasilinear preferences, $R=0$ since the planner attaches the same welfare weight to individuals with the same wage but in different activities, and marginal utility of consumption is constant and equal to one, so that both conditions (ii) and (iv) can be dropped. ${ }^{24}$ Assumptions (i) and (iii) are easy to verify ex-post, as we do in Section 5.

Unbounded Skill Distribution. Our results do not depend on a bounded skill distribution, but readily extend to the case of an unbounded support. For simplicity, consider quasilinear and isoelastic preferences. ${ }^{25}$ Suppose that $\lim _{w \rightarrow \infty} f_{E}^{\varphi}(w) / f_{E}(w)=x$ with $x \in$ $[0,1]$, that $\chi=\lim _{w \rightarrow \infty} w f_{E}(w) /\left(1-F_{E}(w)\right)$ exists, and that $\lim _{w \rightarrow \infty} \psi_{E}(w) / f_{E}(w)=0$ so that the share of rent-seeking income at the top is well-defined, the wage distribution has a Pareto tail, and the social planner puts zero welfare weight on top earners asymptotically. Then we can use equation (17) to derive the following asymptotic marginal tax rate for $w \rightarrow \infty$ (see Rothschild and Scheuer, 2011, for the details):

$$
\lim _{w \rightarrow \infty} T^{\prime}(y(w))=\frac{\xi \chi x+1+1 / \varepsilon}{\chi+1+1 / \varepsilon} .
$$

Moreover, Lemma 3 and Proposition 3 also go through, so that $0<\xi$ and $\xi \lesseqgtr t_{\text {Pigou }}$ under the same conditions as in the bounded support case.

\section{Numerical Illustration}

In this section, we provide optimal policy simulations for a simple version of our model calibrated to the U.S. in order to quantitatively gauge the divergence between the optimal and Pigouvian correction.

\subsection{Data and Estimation}

Data. Our data source is the Current Population Survey (CPS). We take these data as generated by a (sub-optimal) tax equilibrium and use parametric assumptions and equilibrium restrictions from our model to identify the rent-seeking technology and the un-

\footnotetext{
${ }^{24}$ The only role of condition (ii) in Proposition 3 is to make sure (together with regular welfare weights) that the incentive constraints bind downwards, i.e. $\eta(w) \geq 0$. All that matters for this is that the overall social marginal welfare weights $u_{c}(w) \psi_{E}(w) / f_{E}(w)$ are non-increasing in $w$.

${ }^{25}$ Similar results can be derived for the general case using the asymptotic methods in Saez (2001).
} 
derlying skill distribution. Specifically, we use information on worker earnings and hours to generate a sample of hourly wages for the U.S. working population. ${ }^{26}$ In addition, the CPS provides an industry classification that we use to assign individuals to rent-seeking versus traditional work (see Acemoglu and Autor, 2011, and Ales et al., 2014, for recent related exercises). For the sake of illustration and in the spirit of Lockwood et al. (2015), we associate industries related to finance and law services with rent-seeking and all other industries with traditional work. In other words, we take the observed wage distribution and sectoral choices as generated by a two-sector Roy model. We assume quasilinear isoelastic preferences with elasticity $\varepsilon=0.5$.

Externalities. To pin down the externalities from rent-seeking, we use the numbers presented in Lockwood et al. (2015) for aggregate spillovers by sectors (see their table 3). For finance, they estimate this negative spillover to be $1.4 \%$ of aggregate income, based on French's (2008) comparison of active and passive fund management fees and interpreting the difference as wasted resources to beat the market. For law, their estimate of the negative spillover as a share of aggregate income is $0.2 \%$. This is based on Murphy et al.'s (1991) cross-country regressions that measure the effect of the number of lawyers per capita on GDP. The income shares of finance and law in Lockwood et al.'s (2015) calculations are $4.3 \%$ and $2.1 \%$, respectively (see their table 2). Taking these numbers together, we can estimate the negative externalities and hence the Pigouvian correction from the ratio of social and private outputs of these two sectors combined:

$$
1-t_{\text {Pigou }}=\frac{(4.3 \%-1.4 \%)+(2.1 \%-0.2 \%)}{4.3 \%+2.1 \%}=75 \% .
$$

According to this estimate, each dollar of privately earned income in the rent-seeking activity corresponds to only 75 cents of social output, so $t_{\text {Pigou }}=25 \%$. This may be viewed as a conservative estimate of the externalities, though, because (i) French's (2008) analysis, for example, is based on one particular channel of rent-seeking, ignoring others (such as high-speed trading), and (ii) it measures the average externality, which is naturally smaller than the relevant, marginal one (for instance when rent-seeking is associated with crowding effects). We therefore also computed results for the case of twice as large negative externalities, with $t_{\text {Pigou }}=50 \%$. These are shown in Appendix D.

Relative Return Effects. We return to the simple parametrization from Section 2 with constant return elasticities, i.e. $r_{\theta}(E)=k_{\theta} E^{-\beta_{\theta}}$ and $r_{\varphi}(E)=k_{\varphi} E^{-\beta_{\varphi}}$. This is general

\footnotetext{
${ }^{26}$ For further details on the data and sample selection, see Appendix D.
} 
enough to accommodate various scenarios for relative return effects, for any given $t_{\text {Pigou }}$, since $\Delta=\beta_{\varphi}-\beta_{\theta}$ is a free parameter. However, the two parameters $\beta_{\theta}$ and $\beta_{\varphi}$ are jointly constrained by equation (2), because of the Pigouvian correction $t_{\text {Pigou }}$ estimated above and because the income share of the rent-seeking sector $s=Y_{\varphi} / Y$ is given by the data.

This allows us to illustrate the full range of possible relative return effects. At one extreme, if the externality from rent-seeking only falls on the returns to rent-seeking itself, but not on the returns to traditional work, we have $\beta_{\theta}=0$ and, by (2), $\beta_{\varphi}=t_{\text {Pigou }}$. At the other extreme, if the negative spillovers from rent-seeking are borne exclusively by the traditional sector, then $\beta_{\varphi}=0, \beta_{\theta}=s t_{\text {Pigou }} /(1-s)$. Third, in the knife-edge case where both return elasticities are the same, (2) implies $\beta_{\theta}=\beta_{\varphi}=s t_{\text {Pigou }}$ and there are no relative return effects. This parallels the benchmark case that Piketty et al. (2014) and Lockwood et al. (2015) focus on. Finally, we also consider the two intermediate cases, where the effect of $E$ on rent-seeking returns is twice as large as on traditional returns, i.e. $\beta_{\varphi}=2 \beta_{\theta}$, and vice versa, again always consistent with $t_{\text {Pigou }}=25 \%$. This captures situations where the negative spillovers from rent-seeking are borne by both sectors, but more by one or the other.

Estimating the Skill Distribution. The two-dimensional skill distribution $F(\theta, \varphi)$ is estimated as follows. $\theta$ and $\varphi$ are drawn from the sector-specific distributions $F_{\theta}$ and $F_{\varphi}$, which we specify below. Then the correlation is captured by a Gaussian copula, so

$$
F(\theta, \varphi)=N_{\Sigma}\left(\Phi^{-1}\left(F_{\theta}(\theta)\right), \Phi^{-1}\left(F_{\varphi}(\varphi)\right)\right)
$$

where $N_{\Sigma}$ is the cdf of the bivariate normal distribution with mean zero and covariance matrix $\Sigma$, and $\Phi$ is the cdf of the one-dimensional standard normal distribution. In particular, $\Sigma=(1, \rho ; \rho, 1)$, so $\rho$ captures the correlation across the two dimensions of ability, while the marginal distributions are still given by $F_{\theta}$ and $F_{\varphi}$.

Following Lockwood et al. (2015), we assume that these marginals are each described by a Pareto-lognormal distribution. This three-parameter distribution, first introduced by Colombi (1990), approximates a lognormal distribution with mean $\mu_{i}$ and standard deviation $\sigma_{i}$ at low skill values and features a Pareto tail with parameter $\alpha_{i}$ for high skill values, $i \in\{\theta, \varphi\}$. A special case occurs when $\alpha_{i} \rightarrow \infty$, so the marginals become pure lognormal distributions, and hence (26) collapses to a simple bivariate lognormal distribution over $(\theta, \varphi)$. More generally, however, this specification is flexible enough to allow for thicker tails at the top, as implied by the Pareto distribution, which are characteristic of empirical wage and income distributions. 
To estimate the resulting 7 parameters of the bivariate skill distribution (26) (i.e., $\mu_{i}, \sigma_{i}$ and $\alpha_{i}$ for $i \in\{\theta, \varphi\}$ as well as $\rho$ ), we proceed as follows. First, it is straightforward to see that, without loss of generality, we can normalize $E$ and the technological constants $k_{\theta}$ and $k_{\varphi}$ so that $r_{\theta}(E)=r_{\varphi}(E)=1$ at the allocation in the data (see Appendix D for details). As a result, we can take the observed wages in the data as equal to the individuals' skill in the chosen sector: $w=\max \{\theta, \varphi\}$. Based on the wage distribution and sectoral choices observed in the CPS, we then estimate the parameters using a standard two-step Generalized Method of Moments (GMM) procedure. In particular, we compute the percentiles of the overall wage distribution in the data, and then minimize the (weighted) distance between the estimated and empirical share of individuals in each percentile and in each of the two sectors. Appendix D provides further details and the estimation results. It also illustrates the quality of fit between the empirical and estimated sectoral wage distributions based on the 2014 CPS (Figure 2).

\subsection{Results}

Marginal Tax Rate Schedules. Figure 1 shows the optimal marginal income tax schedule, as a function of the hourly wage, for each of the five scenarios for relative return effects described above. The darkest schedule captures the case where the externalities are borne exclusively by rent-seeking $\left(\Delta=t_{\text {Pigou }}>0\right)$, and the schedules are depicted brighter as $\Delta$ becomes smaller (and ultimately negative), always consistent with $t_{\text {Pigou }}=$ $25 \%$. We use relative Pareto weights $\Psi(F)=1-(1-F)^{r}$, where $r=1$ (together with quasilinear preferences) implies the absence of redistributive motives whereas $r \rightarrow \infty$ converges to a Rawlsian criterion. The left panel shows the optimal policies for $r=1$, i.e. utilitarian Pareto weights $\Psi=F$. This captures the benchmark where the income tax purely serves corrective purposes and, by Proposition 2, is given simply by $T^{\prime}(y(w))=$ $\xi f_{E}^{\varphi}(w) / f_{E}(w)$. The right panel shows the optimal marginal tax rates for an intermediate value $r=1.3$, which adds progressive redistributive motives.

The share of rent-seekers is increasing in $w$ for most of the wage distribution and converges to 1 for very high wages given the estimated skill distribution. In the knifeedge scenario with $\beta_{\theta}=\beta_{\varphi}$ and hence no relative return effects, the marginal tax rate schedule is therefore simply $t_{\text {Pigou }} f_{E}^{\varphi}(w) / f_{E}(w)$. This is precisely the middle schedule in the left panel. ${ }^{27}$ In particular, the top marginal tax rate in this scenario is just $t_{\text {Pigou }}$. The other schedules illustrate the consequences of non-trivial relative return effects. The first

\footnotetext{
${ }^{27}$ Recall that $\beta_{\theta}$ and $\beta_{\varphi}$ are chosen to be consistent with $t_{\text {Pigou }}=25 \%$ in the baseline allocation, i.e. given equation (2) and the share of aggregate rent-seeking income $s$ in the data. Since this share is endogenous, however, the Pigouvian correction $t_{\text {Pigou }}$ at the optimum can be slightly different.
} 

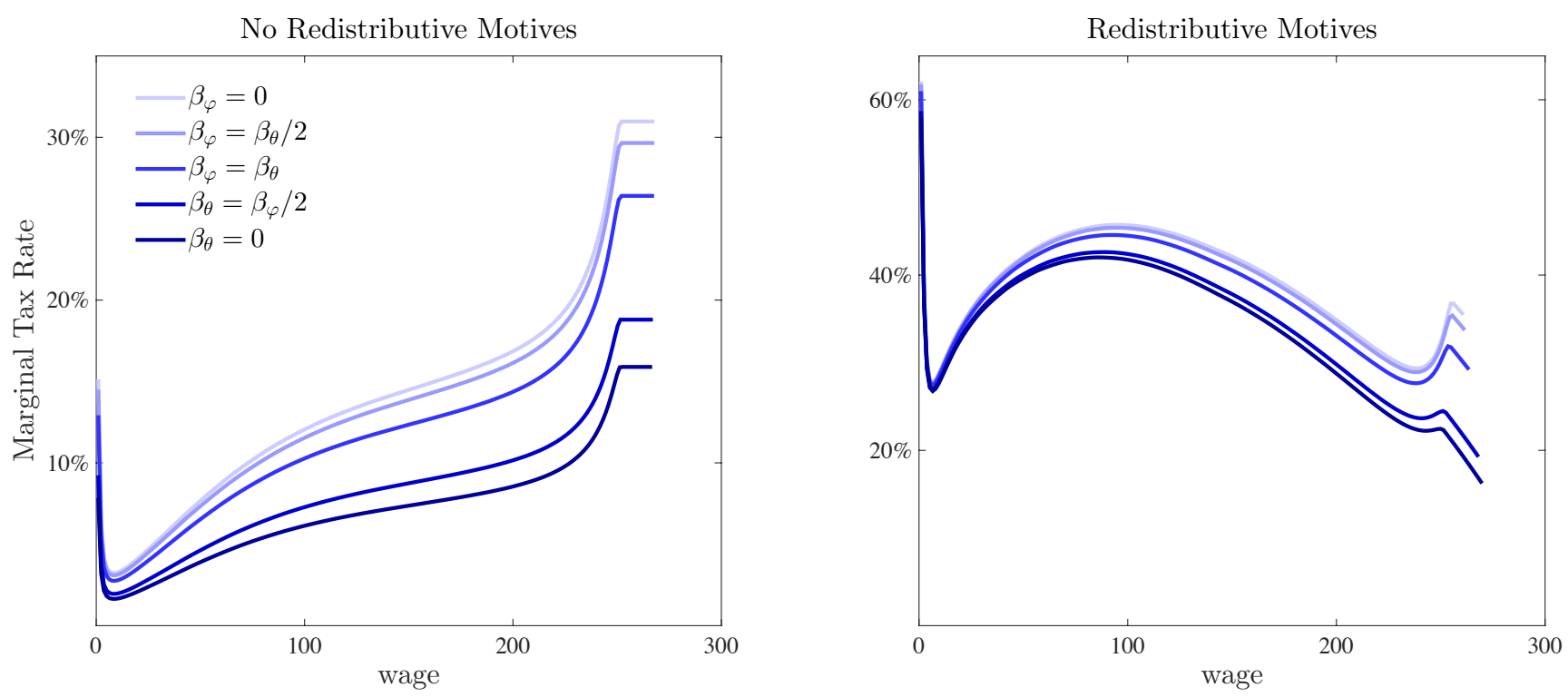

Figure 1: Optimal policy for $t_{\text {Pigou }}=25 \%$, various scenarios

row of Table 1 collects the resulting values for the optimal correction $\xi$, which coincides with the top marginal tax rates in the left panel of Figure 1.

Optimal versus Pigouvian Corrections. As can be seen from Table 1, assuming that the externalities are borne within the rent-seeking sector lowers the optimal correction to $15.9 \%$, i.e. by more than a third compared to the Pigouvian correction. Even in the less extreme and perhaps more plausible scenario where both sectors are affected but the effect on rent-seeking returns is twice as large as on traditional returns, $\xi=18.8 \%$ and thus still considerably lower than in the case without relative return effects. In other words, the activity shift effects $S, C>0$ emphasized here induce a sizable divergence between the optimal correction and what a simple Pigouvian intuition would suggest, indicating that these effects can be of first-order importance, and of similar order of magnitude as the Pigouvian correction itself. ${ }^{28}$

On the other hand, if rent-seeking mostly affects the traditional sector and therefore increases the relative returns to rent-seeking, then this leads to higher-than-Pigouvian taxes in accordance with Proposition 3, up to a top marginal tax rate of $31 \%$. In sum, the magnitude and direction of relative return effects alone, holding the externality as captured by $t_{\text {Pigou }}$ fixed, can lead to a variation in the optimal correction by a factor of 2 when comparing the most extreme cases $(15.9 \%$ versus $31 \%)$, or by a factor of 1.5 when compar-

\footnotetext{
${ }^{28}$ Note that $I=R=0$ in the absence of redistributive motives. It is easy to check numerically that effort $l(w)$ is increasing and $C>0$. This also implies that $y(w)$ is increasing, so that bunching is not part of the optimum.
} 
Table 1: Optimal $\xi$ for different redistributive and technological scenarios

\begin{tabular}{lccccc}
\hline & $\beta_{\theta}=0$ & $\beta_{\theta}=\beta_{\varphi} / 2$ & $\beta_{\theta}=\beta_{\varphi}$ & $\beta_{\varphi}=\beta_{\theta} / 2$ & $\beta_{\varphi}=0$ \\
\hline No redistribution & $15.9 \%$ & $18.8 \%$ & $26.4 \%$ & $29.6 \%$ & $31 \%$ \\
Redistribution & $14.7 \%$ & $17.8 \%$ & $28 \%$ & $32.6 \%$ & $34.4 \%$ \\
\hline
\end{tabular}

ing the more conservative cases where the externalities target both sectors, even though unequally $(29.6 \%$ versus $18.8 \%)$.

Redistributive Motives. The right panel of Figure 1 shows the optimal marginal tax schedules under progressive Pareto weights, with the typical U-shaped pattern for low and intermediate wages and overall degreasing marginal tax rates at the top. The kink at the top occurs at a wage level where the share of rent-seekers hits $100 \%$, and hence the corrective component of the marginal tax rate first increases and then stays flat as wages increase. We observe that redistributive motives further increase the quantitative importance of the relative return effects, as can be seen from the second row of Table 1. The reason is that redistributive goals imply $I>0$ and hence, by equation (24), generate yet another force that drives a wedge between the optimal and Pigouvian correction. This indicates that accounting for redistribution can have further quantitatively significant effects compared to purely efficiency-based approaches, such as Diamond (1973) in the different context of Ramsey commodity taxation, or Lockwood et al. (2015).

Finally, in Appendix D, we present sensitivity checks with respect to the magnitude of $t_{\text {Pigou }}$, and show results for $t_{\text {Pigou }}=50 \%$, i.e., twice the size of the externalities from rentseeking considered here. Unsurprisingly, this also amplifies the optimal divergence from the Pigouvian rate in the considered scenarios. For instance, in the first scenario without cross-sectoral externalities, the optimal correction is $23.7 \%$ without redistributive motives, and $21.6 \%$ with redistributive motives, i.e., less than half the Pigouvian correction (see Figure 3 and Table 2). ${ }^{29}$ In the opposite scenario without within-sector externalities, the optimal corrections are $62.3 \%$ and $72.6 \%$, respectively.

\footnotetext{
${ }^{29}$ For readers who still consider $t_{\text {Pigou }}=50 \%$ as a conservative estimate of the negative spillovers from finance and law, we also computed the optimum for $t_{\text {Pigou }}=90 \%$ and the first scenario, which leads to $\xi=42.5 \%$. Thus, even if the share of rent-seekers at the top is very high and their social marginal product very low, this does not necessarily imply a correspondingly high top marginal tax rate.
} 


\section{Conclusion and Discussion}

Our results are driven by the fact that income taxes are an imperfect instrument for correcting rent-seeking externalities. Directly taxing the externality-causing rent-seeking activity, were it possible, would reduce both its absolute desirability and its desirability relative to other activities. By contrast, an income tax directly affects only the absolute desirability of rent-seeking. The magnitude of the optimal correction via the income tax depends, however, on the direction of the indirect (general equilibrium) effects of taxes on the relative desirability of rent-seeking. When within-activity externalities dominate, these indirect effects are perverse: higher taxes on portions of the income distribution with high levels of rent-seeking raise the relative returns to rent-seeking and encourage a shift towards these activities. Consequently, the optimal externality correction lies strictly below the Pigouvian correction. When across-activity externalities dominate, the indirect effects reinforce the direct effects, and the optimal correction strictly exceeds the Pigouvian one.

We conclude by discussing a few additional considerations that may be important in policy applications.

Information Requirements. We demonstrated the potential quantitative importance of general equilibrium effects and, more generally, how our analytical results can be operationalized to help inform the optimal design of income tax systems. Operationalizing our results requires taking a stand on (i) the magnitude of the rent-seeking externalities in the economy - and the distribution of rent-seeking across income levels—and (ii) the relative impact of these externalities on rent-seeking versus traditional activities. Our results suggest that both the level and relative impacts of rent-seeking externalities are likely to be quantitatively important. These are not well-known or easy-to-obtain quantities though: We know little or nothing about the relative impacts of rent-seeking externalities, and, while we benchmarked the overall magnitude of the externalities to the literature, substantial additional research is needed to pin it down with a higher degree of confidence. We view our analysis as pointing to the importance of such research for income tax policy, similar, for instance, to the work by economists and scientists on the magnitude and distributional impacts of environmental externalities.

Taste Heterogeneity. In our model, individuals' effort choices are driven exclusively by pecuniary concerns. In the full-specialization model described in the main text, for instance, individuals choose whether to be rent-seekers or traditional workers based purely on the wages they can earn in those two sectors. It is straightforward, however, to introduce an extra "distaste for rent-seeking effort" parameter in our framework. If, per 
Lockwood and Weinzierl (2015), this parameter affects preferences over effort and consumption in the same way as skills do, then these two parameters can be collapsed into a single "effective skill" parameter to which all of our analysis directly applies. ${ }^{30}$ As Lockwood and Weinzierl emphasize, however, one needs, in this case, to be careful in how one interprets Pareto weights over these effective skills: if, e.g., a primary driver of income differences is differences in the distaste-for-rent-seeking parameter-with lowdistaste individuals earning high incomes in rent-seeking and high-distaste individuals earning lower incomes in traditional work-one might want to employ more strongly progressive Pareto weights than if differences were driven by skill alone.

Targeting. Our starting point is the assumption that rent-seeking income cannot be directly targeted, which is, of course, what makes our theoretical analysis non-trivial. Our results for this natural benchmark case can be interpreted as a cautionary assessment of the ability of income taxes to substitute for targeted taxes, even if one could perfectly pinpoint the incomes at which rent-seeking predominates.

The extent to which the imperfect targeting assumption is justified differs somewhat across applications. In our calibrated model in Section 5, it means that tax instruments are restricted not to be occupation-specific. This may be because differential taxation creates additional distortions if individuals can relabel their occupations, because it encourages special interest lobbying for preferential tax treatment of particular occupations, or because it raises concerns about horizontal equity and about empowering the government to make the determination of how socially productive workers in different professions are. In other applications, for instance when individuals simultaneously pursue both traditional and rent-seeking effort-as in our Ramsey model-it is even more natural to assume that individuals' total incomes but not their rent-seeking shares are observable, because the government would otherwise be required to collect detailed information about the kind of tasks that individuals do, which is likely difficult and costly.

Regulation. We take the rent-seeking opportunities and hence externalities as given. Presumably, the government can also affect rents through regulation, which would affect the form of the externalities through our technological return functions $r_{\theta}(E)$ and $r_{\varphi}(E)$ directly. However, as long as some rent-seeking opportunities remain after regulation, our analysis of optimal taxes applies, taking the degree of regulation as given.

\footnotetext{
${ }^{30}$ This sort of heterogeneity in the disutility from effort in different sectors is natural in our intensiveeffort-margin Mirrleesian setting. This is different from the sort of fixed costs, unrelated to effort, considered in Lockwood et al. (2015)'s extensive margin model.
} 


\section{References}

Acemoglu, D., and D. Autor (2011): "Skills, Tasks and Technologies: Implications for Employment and Earnings," in Handbook of Labor Economics, ed. by D. Card, and O. Ashenfelter. Elsevier.

Acemoglu, D., and T. Verdier (1998): "Property Rights, Corruption and the Allocation of Talent: A General Equilibrium Approach," Economic Journal, 108, 1381-1403.

Ales, L., M. Kurnaz, and C. Sleet (2015): “Tasks, Talents, and Taxes," American Economic Review, 105, 3061-3101.

Baker, G. (1992): "Incentive Contracts and Performance Measurement," Journal of Political Economy, 100, 98-114.

Baumol, W. (1990): "Entrepreneurship: Productive, Unproductive, and Destructive," Journal of Political Economy, 98, 893-921.

Beaudry, P., C. Blackorby, and D. Szalay (2009): "Taxes and Employment Subsidies in Optimal Redistribution Programs," American Economic Review, 99, 216-242.

Bertrand, M., and S. Mullainathan (2001): “Are CEOs rewarded for luck?," Quarterly Journal of Economics, 116, 901-932.

Besley, T., and M. Ghatak (2013): "Bailouts and the Optimal Taxation of Bonus Pay," American Economic Review, Papers and Proceedings, 103, 163-167.

Cahuc, P., and E. Challe (2012): "Produce or Speculate? Asset Bubbles, Occupational Choice and Efficiency," International Economic Review, 53, 1105-1131.

Choné, P., and G. Laroque (2010): "Negative Marginal Tax Rates and Heterogeneity," American Economic Review, 100, 2532-2547.

Clarke, F. (1976): "A New Approach to Lagrange Multipliers," Mathematics of Operations Research, 1, 165-174.

Colombi, R. (1990): “A New Model of Income Distributions: The Pareto Lognormal Distribution," in Income and Wealth Distribution, Inequality and Poverty, ed. by C. Dagum, and M. Zenga, pp. 18-32. Springer, Berlin.

Cremer, H., F. Gahvari, and N. Ladoux (1998): “Externalities and Optimal Income Taxation," Journal of Public Economics, 70, 343-364.

Diamond, P. (1973): "Consumption Externalities and Imperfect Corrective Pricing," Bell Journal of Economics and Management Science, 4, 526-538.

Diamond, P. (1998): "Optimal Income Taxation: An Example with a U-shaped Pattern of Optimal Tax Rates," American Economic Review, 88, 83-95. 
Dixit, A. (1985): “Tax Policy in Open Economies," in Handbook of Public Economics, ed. by A. Auerbach, and M. Feldstein, pp. 2475-2490. North Holland, Amsterdam.

French, K. (2008): “The Cost of Active Investing," Journal of Finance, 63, 1537-1573.

Fudenberg, D., and J. Tirole (1991): Game Theory. MIT Press, Cambridge.

Golosov, M., P. Maziero, and G. Menzio (2013): “Taxation and Redistribution of Residual Income Inequality," Journal of Political Economy, 121, 1160-1204.

Heathcote, J., F. Perri, and G. Violante (2010): “Unequal we stand: An empirical analysis of economic inequality in the United States, 1967-2006," Review of Economic Dynamics, $13,15-51$.

Holmström, B., and P. Milgrom (1991): "Multitask Principal-Agent Analyses: Incentive Contracts, Asset Ownership, and Job Design," Journal of Law, Economics, and Organization, 7, 24-52.

Hungerbuhler, M., E. Lehmann, A. Parmentier, and B. Linden (2008): “Optimal redistributive taxation in a search equilibrium model," Review of Economic Studies, 73, 743-767.

Kleven, H. J., C. T. Kreiner, and E. Saez (2009): "The Optimal Income Taxation of Couples," Econometrica, 77, 537-560.

Kopczuk, W. (2003): "A Note on Optimal Taxation in the Presence of Externalities," Economics Letters, 80, 81-86.

Lockwood, B., C. Nathanson, and G. Weyl (2015): "Taxation and the Allocation of Talent," Mimeo, Harvard University and University of Chicago.

Lockwood, B., and M. Weinzierl (2015): "De Gustibus non est Taxandum: Heterogeneity in preferences and optimal redistribution," Journal of Public Economics, 124, 74-80.

Mirrlees, J. (1971): “An Exploration in the Theory of Optimum Income Taxation," Review of Economic Studies, 38, 175-208.

Murphy, K., A. Shleifer, and R. Vishny (1991): "The Allocation of Talent: Implications for Growth," Quarterly Journal of Economics, 106, 503-530.

Philippon, T. (2010): "Financiers versus Engineers: Should the Financial Sector be Taxed or Subsidized?," American Economic Journal: Macroeconomics, 2, 158-182.

Piketty, T., E. Saez, and S. Stantcheva (2014): “Optimal Taxation of Top Labor Incomes: A Tale of Three Elasticities," American Economic Journal: Economic Policy, 6, 230271.

Ramsey, F. (1927): "A Contribution to the Theory of Taxation," Economic Journal, 37, 47-61.

Rochet, J.-C., and P. Choné (1998): “Ironing, Sweeping and Multidimensional Screen- 
ing," Econometrica, 66, 783-826.

Rothschild, C., and F. Scheuer (2011): “Optimal Taxation with Rent-Seeking," NBER Working Paper 17035.

(2013): "Redistributive Taxation in the Roy Model," Quarterly Journal of Economics, 128, 623-668.

- (2014): "A Theory of Income Taxation under Multidimensional Skill Heterogeneity," Mimeo, Wellesley College and Stanford University.

Roy, A. (1951): "Some Thoughts on the Distribution of Earnings," Oxford Economic Papers, 3, 135-146.

Sadka, E. (1978): “On the Optimal Taxation of Consumption Externalities," Quarterly Journal of Economics, 92.

Saez, E. (2001): "Using Elasticities to Derive Optimal Tax Rates," Review of Economic Studies, 68, 205-229.

Saez, E., J. Slemrod, and S. Giertz (2012): "The Elasticity of Taxable Income with Respect to the Marginal Tax Rate," Journal of Economic Literature, 50.

Sandmo, A. (1975): "Optimal taxation in the presence of externalities," The Swedish Journal of Economics, 77, 86-98.

Scheuer, F. (2013): “Adverse Selection in Credit Markets and Regressive Profit Taxation," Journal of Economic Theory, 148, 1333-1360.

(2014): "Entrepreneurial Taxation with Endogenous Entry," American Economic Journal: Economic Policy, 6, 126-163.

Stiglitz, J. (1982): "Self-Selection and Pareto Efficient Taxation," Journal of Public Economics, 17, 213-240.

Werning, I. (2000): “An Elementary Proof of Positive Optimal Marginal Tax Rates," Mimeo, MIT. (2007): “Pareto-Efficient Income Taxation," Mimeo, MIT.

\section{A Appendix: Proofs for Section 2}

While we treat the elasticities $\beta_{\theta}, \beta_{\varphi}$, and $\sigma$ as constants in the main text, it will be clear that from the following proofs that the results extend trivially to the case where all three depend on $E$ (in which case the regularity condition $\sigma \Delta>-1+\beta_{\theta}$ must hold uniformly for all relevant $E$ ). The following proofs only rely on $m\left(e_{\theta}, e_{\varphi}\right)$ being linear-homogeneous and weakly quasiconvex. 


\section{A.1 Proof of Lemma 1}

We first show that there is a unique equilibrium $\left(t(E), T(E), E, E_{\theta}(E)\right)$ associated with any $E>0$. (This step does not depend on the regularity condition $\sigma \Delta>-1+\beta_{\theta}$.) Second, we show that the regularity condition implies the co-monotonicity, with $E$, of $E_{\theta}, l=m\left(E_{\theta}, E_{\varphi}\right)$, and $Y$, and the inverse wage $1 / w_{E}$. The co-monotonicity $1-t$ follows easily: $1-t=-u_{l} /\left(w_{E} u_{c}\right)$ by the consumer's problem (6), and $-u_{l} / u_{c}$ is increasing along any upward sloping locus when leisure and consumption are normal goods.

For the first step, note from the necessary and sufficient first-order conditions of the agent's problem (3) first that

$$
\frac{r_{\theta}(E)}{r_{\varphi}(E)}=\frac{m_{\theta}(x, 1)}{m_{\varphi}(x, 1)}
$$

where $x \equiv E_{\theta} / E, m_{\theta}$ and $m_{\varphi}$ denote the partial derivatives of $m$, and second that

$$
t=1+\frac{u_{l}\left(Y\left(E_{\theta}, E\right), m\left(E_{\theta}, E\right)\right) m_{\theta}\left(E_{\theta}, E\right)}{u_{c}\left(Y\left(E_{\theta}, E\right), m\left(E_{\theta}, E\right)\right) r_{\theta}(E)}
$$

Differentiating the log of the left-hand side of (27) with respect to $E$ yields (omitting arguments)

$$
\frac{r_{\theta}}{r_{\varphi}}\left(\frac{r_{\theta}^{\prime}}{r_{\theta}}-\frac{r_{\varphi}^{\prime}}{r_{\varphi}}\right)=\frac{r_{\theta}}{r_{\varphi}} \Delta / E .
$$

Differentiating the log of the right-hand side w.r.t. $x$ yields

$$
\frac{1}{x} \frac{m_{\theta}}{m_{\varphi}}\left(\frac{m_{\theta \theta} x}{m_{\theta}}-\frac{m_{\theta \varphi} x}{m_{\varphi}}\right)=\frac{m_{\theta}}{m_{\varphi}} \frac{1}{\sigma x}>0,
$$

where we used the definition of the substitution elasticity $\sigma=d \log x / d \log \left(m_{\theta} / m_{\varphi}\right)$. If $\Delta=0$, the lefthand-side of (27) is constant, so (27) determines a unique $x$, and $E_{\theta}=E x$. If $\Delta \neq 0$, then, from (29) and (30),

$$
\frac{d x}{d E} \frac{E}{x}=\Delta \sigma .
$$

Hence, (27) implies a unique solution $x(E)$ and $E_{\theta}(E) \equiv E x(E)$ for any $E$. Substituting $E_{\theta}(E)$ for $E_{\theta}$ in (28) yields the unique tax $t(E)$ consistent with an equilibrium with $E$. Budget balance pins down the unique associated transfer $T(E)$. This completes the first step.

For the second step, note that $E_{\theta}=x E$. By (31), $\frac{d E_{\theta}}{d E} \frac{E}{E_{\theta}}=1+\Delta \sigma>1+\Delta \sigma-\beta_{\theta}>0$, so $E_{\theta}$ and $E$ are co-monotonic. Similarly, $l=m\left(E_{\theta}, E\right)=E m(x, 1)$. Hence,

$$
\frac{d l}{d E} \frac{E}{l}=1+\frac{m_{\theta}(x, 1) x}{m(x, 1)} \frac{d x}{d E} \frac{E}{x}=1+\frac{m_{\theta}(x, 1) x}{m(x, 1)} \Delta \sigma .
$$

If $\Delta \geq 0$, this is clearly positive. Otherwise, it exceeds $1+\Delta \sigma>0$ since

$$
\frac{m_{\theta}(x, 1) x}{m(x, 1)}=\frac{m_{\theta}(x, 1) x}{x m_{\theta}(x, 1)+m_{\varphi}(x, 1)} \in(0,1) .
$$

Again similarly, $Y_{\theta}=k_{\theta} E_{\theta} E_{\varphi}^{-\beta_{\theta}}$, so

$$
\frac{d Y_{\theta}}{d E} \frac{E}{Y_{\theta}}=1+\Delta \sigma-\beta_{\theta}>0
$$

Since $Y=Y_{\theta}+k_{\varphi} E^{1-\beta}, Y$ and $E$ are co-monotonic. Finally, $w_{E}$ is the maximum over $x$ of $\left(r_{\theta}(E) x+\right.$ 
$\left.r_{\varphi}(E)\right) / m(x, 1)$, which is decreasing in $E$ (since the returns $r_{\theta}, r_{\varphi}$ are decreasing in $E$ at each $x$ ). So $1 / w_{E}$ is co-monotonic with $E$.

\section{A.2 Proof of Proposition 1}

Using the formulas in the proof of Lemma 1, it is straightforward to show that $E \rightarrow 0$ implies $l \rightarrow 0, Y \rightarrow 0$, and $1 / w_{E} \rightarrow 0$. We can also use Lemma 1 to write the planner's problem in terms of $l$ as $\max _{l} u(Y(l), l)$. The planner's objective is increasing/locally constant/decreasing in $l$ if and only if (suppressing arguments)

$$
u_{c}\left(\frac{d Y}{d l}+\frac{u_{l}}{u_{c}}\right) \gtreqless 0 .
$$

Using $w_{E}=Y / l$ yields

$$
w_{E} u_{c}\left(\frac{d Y}{d l} \frac{l}{Y}+\frac{1}{w_{E}} \frac{u_{l}}{u_{c}}\right) \gtreqless 0 .
$$

Next, write $Y(l)=Y(E(l) x(E(l)), E(l))$ and compute

$$
\begin{aligned}
\frac{d Y}{d l} \frac{Y}{l} & =\left(\frac{d Y}{d E} \frac{E}{Y}\right)\left(\frac{d E}{d l} \frac{l}{E}\right)=\left(\frac{\partial Y}{\partial E}+\frac{\partial Y}{\partial E_{\theta}}\left(E \frac{d x}{d E}+x\right)\right) \frac{E}{Y}\left(\frac{d E}{d l} \frac{l}{E}\right) \\
& =\left(\left(1-t_{\text {Pigou }}\right) r_{\varphi} \frac{E}{Y}+(1-s) \frac{Y}{E_{\theta}} x(1+\Delta \sigma) \frac{E}{Y}\right)\left(1+\frac{m_{\theta}(x, 1) x}{m(x, 1)} \Delta \sigma\right)^{-1} \\
& =\left(\left(1-t_{\text {Pigou }}\right) s+(1-s)(1+\Delta \sigma)\right)(1+(1-s) \Delta \sigma)^{-1} \\
& =\left(s\left(1-\beta_{\varphi}\right)+(1-s)\left(1+\Delta \sigma-\beta_{\theta}\right)\right)(1+(1-s) \Delta \sigma)^{-1}
\end{aligned}
$$

where we used $\partial Y / \partial E_{\theta}=r_{\theta}=(1-s) Y / E_{\theta}$, the definition of $t_{\text {Pigou }}$ in (2), expressions (31) and (33), and the fact that $w_{E} m_{\theta}(x, 1)=r_{\theta}$ from the agent's problem.

Combining (36) and (35) shows that the planner's objective is increasing/locally constant/decreasing in $l$ as

$$
\left[s\left(1-\beta_{\varphi}\right)+(1-s)\left(1+\Delta \sigma-\beta_{\theta}\right)\right](1+(1-s) \Delta \sigma)^{-1} \gtreqless-\frac{1}{w_{E}} \frac{u_{l}}{u_{c}} .
$$

Since $1 / w_{E} \rightarrow 0$ and (by assumption) $-u_{l} / u_{c} \rightarrow 0$ as $E \rightarrow 0$, while

$$
s\left(1-\beta_{\varphi}\right)+(1-s)\left(1+\Delta \sigma-\beta_{\theta}\right) \geq \min \left\{1-\beta_{\varphi}, 1+\Delta \sigma-\beta_{\theta}\right\}>0
$$

and $(1+(1-s) \Delta \sigma) \in(0,1+|\Delta \sigma|)$, we see that the planner's objective is increasing in $l$ as $l \rightarrow 0$. We can therefore find a sufficiently small $l_{0}$ such that the planer's objective is increasing in $l$ at $l_{0}$. We next show that there exists an $l_{1}$ such that the planner's objective $u(Y(l), l)<u\left(Y\left(l_{0}\right), l_{0}\right) \forall l>l_{1}$. The existence of an optimum will then follow from the facts that $\left[l_{0}, l_{1}\right]$ is compact and the equilibrium locus and the planner's objective are continuous.

Towards constructing $l_{1}$, consider the agent's indifference curve in $(l, c)$-space through $\left(l_{0}, Y_{0}\right)$. It is strictly convex and has slope $-u_{l}\left(Y\left(l_{0}\right), l_{0}\right) / u_{c}\left(Y\left(l_{0}\right), l_{0}\right)>0$ at $\left(l_{0}, Y\left(l_{0}\right)\right)$. Hence, along the indifference curve, the ratio $c / l$ is bounded below by $-u_{l}\left(Y\left(l_{0}\right), l_{0}\right) / u_{c}\left(Y\left(l_{0}\right), l_{0}\right)>0$ as $(l, c)$ grows large. On the other hand, the ratio $Y(l) / l=w(l) \rightarrow 0$ along the equilibrium locus. So there exists an $l_{1}$ sufficiently high so that the indifference curve lies strictly above the equilibrium locus.

Finally, to obtain the optimal tax formula (8) and complete the proof, note that a necessary condition for the planner's optimum is that (35) holds with equality. Using (36) and the fact, from the agent's opti- 
mization problem, that $(1-t) w=-u_{l} / u_{c}$, yields

$$
1-t=\left(\left(1-t_{\text {Pigou }}\right) s+(1-s)(1+\Delta \sigma)\right)(1+(1-s) \Delta \sigma)^{-1} .
$$

Rearranging yields (8).

\section{A.3 Details on Section 2.4 and Proof or Corollary 1}

Using the functional forms for the returns and preferences, we can write (27) and (28) as $x=E^{\Delta \sigma}\left(k_{\theta} / k_{\varphi}\right)^{\sigma}$ and

$$
(1-t) k_{\varphi} E^{-\beta_{\varphi}}=e_{\varphi}^{\frac{1}{\varepsilon}}\left(1+x^{\frac{1+\sigma}{\sigma}}\right)^{\frac{\sigma-\varepsilon}{\varepsilon(1+\sigma)}} .
$$

Using $e_{\varphi}=E$ and the first condition to write $E$ as a function of $x$ in the second condition, we obtain

Finally, note that

$$
1-t=k_{\theta}^{-\frac{1+\varepsilon \beta \varphi}{\Delta \varepsilon}} k_{\varphi}^{\frac{1+\varepsilon \beta \varphi-\Delta \varepsilon}{\Delta \varepsilon}} x^{\frac{1+\varepsilon \beta \varphi}{\varepsilon \Delta \sigma}}\left(1+x^{\frac{1+\sigma}{\sigma}}\right)^{\frac{\sigma-\varepsilon}{\varepsilon(1+\sigma)}}
$$

$$
s=\frac{Y_{\varphi}}{Y}=\frac{k_{\varphi} E^{1-\beta_{\varphi}}}{k_{\theta} E_{\theta} E^{-\beta_{\theta}}+k_{\varphi} E^{1-\beta_{\varphi}}}=\left(1+\frac{k_{\theta} x E^{1-\beta_{\theta}}}{k_{\varphi} E^{1-\beta_{\varphi}}}\right)^{-1}=\left(1+\frac{k_{\theta}}{k_{\varphi}} x E^{\Delta}\right)^{-1}=\left(1+x^{\frac{1+\sigma}{\sigma}}\right)^{-1} .
$$

Using this above to substitute $s$ for $x$ delivers equation (10) in the main text, with $K \equiv k_{\theta}^{-\frac{1+\varepsilon \beta \varphi}{\Delta \varepsilon}} k_{\varphi}^{\frac{1+\varepsilon \beta_{\varphi}-\Delta \varepsilon}{\Delta \varepsilon}}$.

To prove Corollary 1 , note that an optimal equilibrium is an intersection of the two functions $t^{\text {opt }}(s)$ and $t^{e q}(s)$, where the former is given by the right-hand side of (9) and the latter is given by 1 minus the right-hand side of (10), for any $s \in[0,1]$.

As for the former, in the limit as $\sigma \rightarrow \infty$, $t^{\text {opt }}(s)$ is identically 0 for $s \in[0,1)$ if $\Delta>0$. Moreover, $t^{o p t}(1)=\beta_{\varphi}$ for all $\sigma$. As for the latter, if $\Delta>0, t^{e q}(s)$ converges on $s \in(0,1)$ to the increasing limit function $\bar{t}^{e q}(s)=1-K s^{-1 / \varepsilon}$. For $s \rightarrow 0, \bar{t}^{e q}(s) \rightarrow-\infty$ and for $s \rightarrow 1, \bar{t}^{e q}(s) \rightarrow 1-K$.

If $K<1-\beta_{\varphi}$, comparing the limit points of the graphs of the functions $\bar{t}^{e q}(s)$ and $\bar{t}^{o p t}(s)$ as $\sigma \rightarrow \infty$ shows that, for sufficiently large $\sigma$, there must be a unique $s(\sigma)$ for which $\bar{t}^{e q}(s(\sigma))=\bar{t}^{e q}(s(\sigma))$, and this unique intersection, which is the optimum, must satisfy $s(\sigma) \rightarrow K^{1 / \varepsilon} \in(0,1)$ and $\bar{t}^{e q}(s(\sigma)) \rightarrow 0$.

\section{B Appendix: Proofs for Section 3}

In this appendix, we show how our analysis from Section 3.2 extends to the case where the effort aggregator $m$ is quasiconvex and linear homogeneous, which allows for the linear $m$ case discussed in the main text as a special case. We again characterize a direct implementation, where individuals announce their privately known type $(\theta, \varphi)$ and then get assigned $c(\theta, \varphi), y(\theta, \varphi)$, and the fraction of income earned through the $\Theta$-activity, given by $q(\theta, \varphi) \equiv y_{\theta}(\theta, \varphi) / y(\theta, \varphi)=r_{\theta}(E) \theta e_{\theta}(\theta, \varphi) / y(\theta, \varphi)$. Income $y$ and consumption $c$ are observable but an individual's skill type $(\theta, \varphi)$ and the activity-specific efforts $e_{\theta}$ or $e_{\varphi}$ (and $q$ ) are not. The 
resulting incentive constraints that guarantee truth-telling of the agents are:

$$
\begin{aligned}
& u\left(c(\theta, \varphi), m\left(\frac{q(\theta, \varphi) y(\theta, \varphi)}{\theta r_{\theta}(E)}, \frac{(1-q(\theta, \varphi)) y(\theta, \varphi)}{\varphi r_{\varphi}(E)}\right)\right) \\
& \quad \geq \max _{p \in[0,1]}\left\{u\left(c\left(\theta^{\prime}, \varphi^{\prime}\right), m\left(\frac{p y\left(\theta^{\prime}, \varphi^{\prime}\right)}{\theta r_{\theta}(E)}, \frac{(1-p) y\left(\theta^{\prime}, \varphi^{\prime}\right)}{\varphi r_{\varphi}(E)}\right)\right)\right\} \forall(\theta, \varphi),\left(\theta^{\prime}, \varphi^{\prime}\right),
\end{aligned}
$$

since type $(\theta, \varphi)$ can imitate another type $\left(\theta^{\prime}, \varphi^{\prime}\right)$ by earning $\left(\theta^{\prime}, \varphi^{\prime}\right)^{\prime}$ s income using a continuum of combinations of efforts (and thus income shares $(p, 1-p)$ ) in the $\Theta$ - and $\Phi$-activities.

The following lemma shows that incentive compatibility implies that each type $(\theta, \varphi)$ has a well-defined wage $w \equiv y / l$ and activity-specific income share $q$, which both depend on aggregate rent-seeking effort $E$ but are otherwise independent of the allocation.

Lemma 4. In any incentive compatible allocation $\{c(\theta, \varphi), y(\theta, \varphi), q(\theta, \varphi), E\}$,

$$
w(\theta, \varphi) \equiv \frac{y(\theta, \varphi)}{l(\theta, \varphi)}=\max _{p \in[0,1]} m\left(\frac{p}{\theta r_{\theta}(E)}, \frac{1-p}{\varphi r_{\varphi}(E)}\right)^{-1}
$$

and $q(\theta, \varphi)$ is a corresponding arg max.

Proof. By the linear homogeneity of $m$, the "own type" incentive constraints (for $(\theta, \varphi)=\left(\theta^{\prime}, \varphi^{\prime}\right)$ ) imply

$$
q(\theta, \varphi) \in \underset{p \in[0,1]}{\operatorname{argmin}} m\left(\frac{p y(\theta, \varphi)}{\theta r_{\theta}(E)}, \frac{(1-p) y(\theta, \varphi)}{\varphi r_{\varphi}(E)}\right)=\underset{p \in[0,1]}{\operatorname{argmin}} m\left(\frac{p}{\theta r_{\theta}(E)}, \frac{1-p}{\varphi r_{\varphi}(E)}\right) .
$$

Equation (40) then follows immediately from the definitions $w(\theta, \varphi) \equiv y(\theta, \varphi) / l(\theta, \varphi)$ and

$$
l(\theta, \varphi) \equiv m\left(e_{\theta}(\theta, \varphi), e_{\varphi}(\theta, \varphi)\right)=y(\theta, \varphi) m\left(\frac{q(\theta, \varphi)}{\theta r_{\theta}(E)}, \frac{1-q(\theta, \varphi)}{\varphi r_{\varphi}(E)}\right) .
$$

By Lemma 4, fixing $E$ pins down each type's wage $w_{E}(\theta, \varphi)$, as in (5). Moreover, $q(\theta, \varphi)$ is chosen to minimize the overall effort $m\left(e_{\theta}, e_{\varphi}\right)$ subject to achieving a given amount of income. When $m$ is strictly quasiconvex, $q(\theta, \varphi)$, which depends only on the skill ratio $\alpha \equiv \theta / \varphi$, is also uniquely determined by $E$ for each $\alpha$. With weakly quasiconvex $m, q(\theta / \varphi)$ is uniquely determined outside of a countable set of $\alpha$-values, where it is interval-valued because the individual is indifferent between various effort combinations to achieve a given income. By Lemma 4, the correspondence $q(\theta / \varphi)$ depends on, and is non-decreasing in, the relative returns $\zeta_{E}(\alpha) \equiv \alpha r_{\theta}(E) / r_{\varphi}(E)$, and we can define the functions $q_{E}(\alpha) \equiv Q\left(\zeta_{E}(\alpha)\right)$ by taking $q$ to be equal to the midpoints of the intervals on the countable set of degenerate $\alpha^{\prime}$ s.

Viewed as a distribution, $Q$ has a well-defined derivative denoted by $Q^{\prime} \cdot{ }^{31}$ Since

$$
x=\frac{e_{\theta}}{e_{\varphi}}=\frac{\varphi r_{\varphi}(E)}{\theta r_{\theta}(E)}\left(\frac{y}{e_{\varphi} \varphi r_{\varphi}(E)}-1\right)=\frac{1}{\zeta_{E}(\alpha)} \frac{q}{1-q},
$$

we can write $Q(\zeta)=\zeta x(\zeta) /(1+\zeta x(\zeta))$, which only depends on and is increasing in $\zeta$ by linear homogeneity and quasiconvexity of $m$. For later use, it will also be helpful to define $\tilde{Q}_{\zeta_{0}}(\zeta) \equiv \zeta_{0} x(\zeta) /\left(1+\zeta_{0} x(\zeta)\right)$ with $\left.\tilde{Q}^{\prime}(\zeta) \equiv \tilde{Q}_{\zeta_{0}}^{\prime}(\zeta)\right|_{\zeta_{0}=\zeta^{\prime}}$ i.e. the change in the traditional income share $Q$ that is due to the change in the activity-specific effort ratio $x$ in response to a change in relative returns $\zeta$, but holding $\zeta$ fixed otherwise.

\footnotetext{
${ }^{31}$ In particular, $Q(\zeta)$ is ordinarily differentiable except at some countable number of jump discontinuities; the latter adds a series of Dirac $\delta$-functions to $Q^{\prime}$. See Appendix C.3. Recall that $q \in\{0,1\}$ for almost all individuals if $m$ is linear, but otherwise will typically take interior values.
} 


\section{Appendix: Proofs for Section 4}

\section{C.1 Wage Distributions for General $m$}

Lemma 4 showed that fixing $E$ determines the wage $w_{E}(\theta, \varphi)$ and the traditional activity income share $q_{E}(\alpha)$ for each type $(\theta, \varphi)$. For any given $E$, we compute the cdf over $(w, \alpha)$-pairs:

$$
G_{E}(w, \alpha) \equiv \int_{\left\{(\theta, \varphi) \mid w_{E}(\theta, \varphi) \leq w, \theta / \varphi \leq \alpha\right\}} d F(\theta, \varphi)
$$

with the corresponding density $g_{E}(w, \alpha)$. This allows us to obtain the wage distribution for any given $E$ simply as the marginal distribution

$$
F_{E}(w) \equiv \int_{\left\{(\theta, \varphi) \mid w_{E}(\theta, \varphi) \leq w\right\}} d F(\theta, \varphi)=\int_{\underline{w}_{E}}^{w} \int_{\alpha=0}^{\infty} d G_{E}(z, \alpha)
$$

with the corresponding density $f_{E}(w)=\int_{\alpha=0}^{\infty} d G_{E}(w, \alpha)$ as well as the activity-specific densities $f_{E}^{\theta}(w) \equiv$ $\int_{\alpha=0}^{\infty} q_{E}(\alpha) d G_{E}(w, \alpha)$ and $f_{E}^{\varphi}(w) \equiv \int_{\alpha=0}^{\infty}\left(1-q_{E}(\alpha)\right) d G_{E}(w, \alpha)$. Hence, these densities can be interpreted as an average value of $q$ (respectively $1-q$ ) for all individuals at wage $w$, and $f_{E}(w)=f_{E}^{\theta}(w)+f_{E}^{\varphi}(w)$ for all $w \in\left[\underline{w}_{E}, \bar{w}_{E}\right]$. In the special case with $m\left(e_{\theta}, e_{\varphi}\right)=e_{\theta}+e_{\varphi},(40)$ immediately implies $q_{E}(\alpha) \in\{0,1\}$ almost everywhere, leading to the definitions in the main text.

\section{C.2 Proof of Proposition 2}

Putting multipliers $\lambda$ on (15), $\xi \lambda$ on (14) and $\hat{\eta}(w) \lambda$ on (13), the Lagrangian corresponding to (12)-(15) is, after integrating by parts (13),

$$
\begin{aligned}
\mathcal{L} & =\int_{\underline{w}_{E}}^{\bar{w}_{E}} V(w) \psi_{E}(w) d w-\int_{\underline{w}_{E}}^{\bar{w}_{E}} V(w) \hat{\eta}^{\prime}(w) \lambda d w+\int_{\underline{w}_{E}}^{\bar{w}_{E}} u_{l}(c(V(w), l(w)), l(w)) \frac{l(w)}{w} \hat{\eta}(w) \lambda d w \\
+ & \xi \lambda \gamma_{\varphi}(E)-\xi \lambda \int_{\underline{w}_{E}}^{\bar{w}_{E}} w l(w) f_{E}^{\varphi}(w) d w+\lambda \int_{\underline{w}_{E}}^{\bar{w}_{E}} w l(w) f_{E}(w) d w-\lambda \int_{\underline{w}_{E}}^{\bar{w}_{E}} c(V(w), l(w)) f_{E}(w) d w .
\end{aligned}
$$

By Theorem 3 in Clarke (1976), this Lagrangian approach is generically valid, i.e. constraint qualification is satisfied and any solution must be a stationary point of Lagrangian for some (bounded) multipliers. Using $\partial c / \partial V=1 / u_{c}$ and compressing notation, the first order condition for $V(w)$ is

$$
\hat{\eta}^{\prime}(w) \lambda=\psi_{E}(w)-\lambda f_{E}(w) \frac{1}{u_{c}(w)}+\hat{\eta}(w) \lambda \frac{u_{l c}(w)}{u_{c}(w)} \frac{l(w)}{w} .
$$

Defining $\eta(w) \equiv \hat{\eta}(w) u_{c}(w)$, this becomes

$$
\eta^{\prime}(w)=\psi_{E}(w) \frac{u_{c}(w)}{\lambda}-f_{E}(w)+\eta(w) \frac{u_{c c}(w) c^{\prime}(w)+u_{c l}(w) l^{\prime}(w)+u_{c l}(w) l(w) / w}{u_{c}(w)} .
$$

Using the first order condition corresponding to the incentive constraint (16),

$$
u_{c}(w) c^{\prime}(w)+u_{l}(w) l^{\prime}(w)+u_{l}(w) \frac{l(w)}{w}=0,
$$


the fraction in (44) can be written as $-(\partial M R S(w) / \partial c) y^{\prime}(w) / w$, where $M(c, l) \equiv-u_{l}(c, l) / u_{c}(c, l)$ is the marginal rate of substitution between effort and consumption and $M R S(w) \equiv M(c(w), l(w)$ ), so (with a slight abuse of notation) $\partial M R S(w) / \partial c$ stands short for $\partial M(c(w), l(w)) / \partial c$. Substituting in (44) and rearranging yields

$$
-\frac{\partial M R S(w)}{\partial c} l(w) \frac{y^{\prime}(w)}{y(w)} \eta(w)=f_{E}(w)-\psi_{E}(w) \frac{u_{\mathcal{c}}(w)}{\lambda}+\eta^{\prime}(w)
$$

Integrating this ODE gives

$$
\begin{aligned}
\eta(w) & =\int_{w}^{\bar{w}_{E}}\left(f_{E}(x)-\psi_{E}(x) \frac{u_{c}(x)}{\lambda}\right) \exp \left(\int_{w}^{x} \frac{\partial M R S(s)}{\partial c} l(s) \frac{y^{\prime}(s)}{y(s)} d s\right) d x \\
& =\int_{w}^{\bar{w}_{E}}\left(1-\frac{\psi_{E}(x)}{f_{E}(x)} \frac{u_{c}(x)}{\lambda}\right) \exp \left(\int_{w}^{x}\left(1-\frac{\varepsilon^{u}(s)}{\varepsilon^{c}(s)}\right) \frac{d y(s)}{y(s)}\right) f_{E}(x) d x
\end{aligned}
$$

where the last step follows from $l(w) \partial M R S(w) / \partial c=1-\varepsilon^{u}(w) / \varepsilon^{c}(w)$ after tedious algebra (e.g. using equations (23) and (24) in Saez, 2001).

Using $\partial c / \partial l=M R S$, the first order condition for $l(w)$ is

$$
\lambda w f_{E}(w)\left(1-\frac{M R S(w)}{w}\right)-\xi \lambda w f_{E}^{\varphi}(w)=-\hat{\eta}(w) \lambda\left[\frac{\left(-u_{l c}(w) u_{l}(w) / u_{c}(w)+u_{l l}(w)\right) l(w)}{w}+\frac{u_{l}(w)}{w}\right]
$$

which after some algebra can be rewritten as

$$
w f_{E}(w)\left(1-\frac{M R S(w)}{w}\right)-\xi w f_{E}^{\varphi}(w)=\eta(w)\left(\frac{\partial M R S(w)}{\partial l} \frac{l}{w}+\frac{M R S(w)}{w}\right)
$$

where $\partial M R S(w) / \partial e$ again stands short for $\partial M(c(w), l(w)) / \partial l$. With $M R S(w) / w=1-T^{\prime}(y(w))$ from the first-order condition of the workers, this becomes

$$
1-\xi \frac{f_{E}^{\varphi}(w)}{f_{E}(w)}=\left(1-T^{\prime}(y(w))\right)\left[1+\frac{\eta(w)}{w f_{E}(w)}\left(1+\frac{\partial M R S(w)}{\partial l} \frac{l}{M R S(w)}\right)\right]
$$

Simple algebra again shows that $1+\partial \log M R S(w) / \partial \log l=\left(1+\varepsilon^{u}(w)\right) / \varepsilon^{c}(w)$, so that the result follows from (47) and (49).

\section{C.3 Proof of Lemma 3}

We prove the following lemma for the case of a general $m$-aggregator function. Lemma 3 will then emerge as a special case for linear $m$.

Lemma 5. The welfare effect of a marginal change in aggregate rent-seeking effort $E$ is given by (19) in Lemma 3. The terms I and $R$ are as in (20) and (21). $C$ and $S$ are given by

$$
C \equiv \int_{\underline{w}_{E}}^{\bar{w}_{E}} w^{2} l^{\prime}(w) \operatorname{Var}_{E}(q \mid w) f_{E}(w) d w
$$

and

$$
S \equiv \int_{\underline{w}_{E}}^{\bar{w}_{E}} \int_{\alpha=0}^{\infty} y(w) \tilde{Q}^{\prime}\left(\zeta_{E}(\alpha)\right) \zeta_{E}(\alpha) d G_{E}(w, \alpha) \geq 0
$$

with $\operatorname{Var}_{E}(q \mid w)=\int_{0}^{\infty} q_{E}(\alpha)^{2} g_{E}(\alpha \mid w) d \alpha-\left(\int_{0}^{\infty} q_{E}(\alpha) g_{E}(\alpha \mid w) d \alpha\right)^{2}$ and $g_{E}(\alpha \mid w)=\frac{g_{E}(w, \alpha)}{f_{E}(w)}$. 
Before proving Lemma 5, we establish some preliminary results. Because we allow $m$ to be only weakly quasiconvex, the marginal rate of substitution $\chi\left(e_{\theta} / e_{\varphi}\right) \equiv m_{\theta}\left(e_{\theta} / e_{\varphi}\right) / m_{\varphi}\left(e_{\theta} / e_{\varphi}\right)$ can have constant regions. Since $\chi$ is non-decreasing, there are at most a countable number of such regions. Use $\left\{d_{i}\right\}_{i=1}^{n}$, with $d_{i}$ increasing in $i$, to denote the values of $\chi$ at these flat regions. ${ }^{32}$ An individual with $\theta / \varphi=\frac{r_{\varphi}(E)}{r_{\theta}(E)} d_{i} \equiv \alpha_{E}^{i}$ will be indifferent between a range of activity-specific income shares $q \in\left[q^{i}, \bar{q}^{i}\right]$, where, by equation (41), the bounds are, respectively, the minimum and the maximum $q$ for which $\chi\left(\frac{q}{1-q} \frac{1}{d_{i}}\right)=d_{i}$ and thus independent of $E$. The upper-hemicontinuous correspondence $q_{E}(\alpha)$ thus jumps from $q^{i}$ to $\bar{q}^{i}$ as $\alpha$ crosses $\alpha_{E}^{i}$ from below. For any given wage $w$ and $E$, taking $\alpha_{E}^{0}=\underline{\theta} / \bar{\varphi}$ and $\alpha_{E}^{n+1}=\bar{\theta} / \underline{\varphi}$, we can thus write ${ }^{33}$

$$
F_{E}(w)=\int_{\alpha_{E}^{0}}^{\alpha_{E}^{n+1}} \int_{\underline{w}_{E}(\alpha)}^{w} g_{E}\left(w^{\prime}, \alpha\right) d w^{\prime} d \alpha=\sum_{i=0}^{n} \int_{\alpha_{E}^{i}}^{\alpha_{E}^{i+1}} \int_{\underline{w}_{E}(\alpha)}^{w} g_{E}\left(w^{\prime}, \alpha\right) d w^{\prime} d \alpha,
$$

where $\underline{w}_{E}(\alpha) \equiv w_{E}(\max \{\underline{\theta}, \alpha \underline{\varphi}\}, \max \{\underline{\theta} / \alpha, \underline{\varphi}\})$ and $\bar{w}_{E}(\alpha) \equiv w_{E}(\min \{\bar{\theta}, \alpha \bar{\varphi}\}, \min \{\bar{\theta} / \alpha, \bar{\varphi}\})$. Similarly, we can write

$$
F_{E}^{\theta}(w)=\sum_{i=0}^{n} \int_{\alpha_{E}^{i}}^{\alpha_{E}^{i+1}} \int_{\underline{w}_{E}(\alpha)}^{w} q_{E}(\alpha) g_{E}\left(w^{\prime}, \alpha\right) d w^{\prime} d \alpha .
$$

The latter is a useful formulation because, on each of the intervals in the sum, the function $q_{E}(\alpha)$ is continuously differentiable. The discontinuities occur at the boundaries of the intervals. It allows us to prove the following technical lemma, which will be useful below.

\section{Lemma 6.}

$$
\begin{gathered}
\frac{d F_{E}(w)}{d E}=\frac{\beta_{\varphi}(E)}{E} w f_{E}^{\varphi}(w)+\frac{\beta_{\theta}(E)}{E} w f_{E}^{\theta}(w) \text { and } \frac{d F_{E}^{\theta}(w)}{d E}=\frac{\beta_{\theta}(E)}{E} w f_{E}^{\theta}(w)-K_{E}(w)-L_{E}(w), \text { where } \\
K_{E}(w) \equiv-\sum_{i=0}^{n} \int_{\alpha_{E}^{i}}^{\alpha_{E}^{i+1}} \int_{\underline{w}_{E}(\alpha)}^{w} \frac{d q_{E}(\alpha)}{d E} g_{E}\left(w^{\prime}, \alpha\right) d w^{\prime} d \alpha+\sum_{i=1}^{n} \int_{\underline{w}_{E}\left(\alpha_{E}^{i}\right)}^{w} \frac{d \alpha_{E}^{i}}{d E}\left(\bar{q}^{i}-q^{i}\right) g_{E}\left(w^{\prime}, \alpha_{E}^{i}\right) d w^{\prime} \\
=-\frac{\Delta(E)}{E} \int_{\alpha_{E}^{0}}^{\alpha_{E}^{n+1}} \int_{\underline{w}_{E}(\alpha)}^{w} Q^{\prime}\left(\zeta_{E}(\alpha)\right) \zeta_{E}(\alpha) g_{E}\left(w^{\prime}, \alpha\right) d w^{\prime} d \alpha \\
\text { and } L_{E}(w) \equiv-\frac{\Delta(E)}{E} \int_{\alpha_{E}^{0}}^{\alpha_{E}^{n+1}} q_{E}(\alpha)\left(1-q_{E}(\alpha)\right) w g_{E}(w, \alpha) d \alpha .
\end{gathered}
$$

Moreover, $d F_{E}^{\varphi}(w) / d E=d F_{E}(w) / d E-d F_{E}^{\theta}(w) / d E$ and analogous expressions hold for $d \Psi_{E}(w) / d E, d \Psi_{E}^{\theta}(w) / d E$ and $d \Psi_{E}^{\varphi}(w) / d E$.

Proof. It is useful to define the function $\tilde{w}\left(w, \alpha, E ; E_{0}\right)$ as the wage, at $E$, of the type $(\theta, \varphi)$ that would have had wage $w$ (and $\alpha$ ) at $E=E_{0}$. Then, by construction, the set $\left\{(\theta, \varphi) \mid w_{E}(\theta, \varphi) \leq \tilde{w}\left(w, \theta / \varphi, E ; E_{0}\right)\right\}$ is independent of $E$ : it is simply the set of types that would have had wage less than $w$ at $E_{0}$. Hence, for all E,

$$
F_{E_{0}}(w)=\int_{\alpha=\alpha_{E}^{0}}^{\alpha_{E}^{n+1}} \int_{\underline{w}_{E}(\alpha)}^{\tilde{w}\left(w, \alpha, E ; E_{0}\right)} g_{E}\left(w^{\prime}, \alpha\right) d w^{\prime} d \alpha
$$

\footnotetext{
${ }^{32}$ We deal with finite $n$ to keep notation clean; the countably infinite case is an easy but notationally cumbersome extension.

${ }^{33}$ We mildly abuse notation here in assuming that $\alpha_{E}^{0} \leq \alpha_{E}^{1}$ and $\alpha_{E}^{n+1} \geq \alpha_{E}^{n}$ since a simple redefinition of $n$ and the relevant $\alpha_{E}^{i}$ values would yield the same formulae.
} 
i.e., the measure of types with wage less than $w$ when $E=E_{0}$. Taking the derivative of the RHS with respect to $E$ (which is zero by construction) and evaluating at $E_{0}$ and re-arranging yields

$$
\int_{\alpha=\alpha_{E}^{0}}^{\alpha_{E}^{n+1}} \int_{\underline{w}_{E}(\alpha)}^{w} \frac{d g_{E_{0}}\left(w^{\prime}, \alpha\right)}{d E} d w^{\prime} d \alpha-\int_{\alpha=\alpha_{E}^{0}}^{\alpha_{E}^{n+1}} \frac{d \underline{w}_{E_{0}}(\alpha)}{d E} g_{E_{0}}\left(\underline{w}_{E_{0}}, \alpha\right) d \alpha=-\int_{\alpha=\alpha_{E}^{0}}^{\alpha_{E}^{n+1}} \frac{d \tilde{w}\left(w, \alpha, E_{0} ; E_{0}\right)}{d E} g_{E_{0}}(w, \alpha) d \alpha .
$$

The LHS is easily recognized as $d F_{E}(w) / d E$, evaluated at $E_{0}$. Computing $d \tilde{w}\left(w, \alpha, E_{0} ; E_{0}\right) / d E$,

$$
\frac{d F_{E}(w)}{d E}=\int_{\alpha=\alpha_{E}^{0}}^{\alpha_{E}^{n+1}}\left(q_{E}(\alpha) \frac{\beta_{\theta}(E)}{E} w+\left(1-q_{E}(\alpha)\right) \frac{\beta_{\varphi}(E)}{E} w\right) g_{E}(w, \alpha) d \alpha=\frac{\beta_{\theta}(E)}{E} w f_{E}^{\theta}(w)+\frac{\beta_{\varphi}(E)}{E} w f_{E}^{\varphi}(w),
$$

which proves the first result in (52). Moreover, for all $E$,

$$
F_{E_{0}}^{\theta}(w)=\sum_{i=0}^{n} \int_{\alpha_{E_{0}}^{i}}^{\alpha_{E_{0}}^{i+1}} \int_{\underline{w}_{E}(\alpha)}^{\tilde{w}\left(w, \alpha, E ; E_{0}\right)} q_{E_{0}}(\alpha) g_{E}\left(w^{\prime}, \alpha\right) d w^{\prime} d \alpha
$$

since the set of types being integrated over is independent of $E$, and so is $q_{E_{0}}(\alpha)$. We explicitly compute the derivative with respect to $E$ of the RHS of (56), which is zero since the object is, by construction, independent of $E$. After some re-arranging and evaluating at $E_{0}$, we get

$$
\begin{aligned}
& \sum_{i=0}^{n} \int_{\alpha_{E_{0}}^{i}}^{\alpha_{E_{0}}^{i+1}} \int_{\underline{w}_{E_{0}}(\alpha)}^{w} q_{E_{0}}(\alpha) \frac{d g_{E_{0}}\left(w^{\prime}, \alpha\right)}{d E} d w^{\prime} d \alpha-\sum_{i=0}^{n} \int_{\alpha_{E_{0}}^{i}}^{\alpha_{E_{0}}^{i+1}} \frac{d \underline{w}_{E_{0}}(\alpha)}{d E} q_{E_{0}}(\alpha) g_{E_{0}}\left(\underline{w}_{E_{0}}(\alpha), \alpha\right) d \alpha \\
& =-\sum_{i=0}^{n} \int_{\alpha_{E_{0}}^{i}}^{\alpha_{E_{0}}^{i+1}} \frac{d \tilde{w}\left(w, \alpha, E_{0} ; E_{0}\right)}{d E} q_{E_{0}}(\alpha) g_{E_{0}}(w, \alpha) d \alpha .
\end{aligned}
$$

Adding $\sum_{i=0}^{n} \int_{\alpha_{E_{0}}^{i}}^{\alpha_{E_{0}}^{i+1}} \int_{\underline{w}_{E_{0}}(\alpha)}^{w} \frac{d q_{E_{0}}(\alpha)}{d E} g_{E_{0}}\left(w^{\prime}, \alpha\right) d w^{\prime} d \alpha-\sum_{i=1}^{n} \int_{\underline{w}_{E_{0}}}^{w}\left(\alpha_{E_{0}}^{i}\right) \frac{d \alpha_{E_{0}}^{i}}{d E}\left(\bar{q}^{i}-\underline{q}^{i}\right) g_{E_{0}}\left(w^{\prime}, \alpha_{E_{0}}^{i}\right) d w^{\prime}$ to both sides, we can recognize the left hand side as $d F_{E}^{\theta}(w) / d E$ evaluated at $E_{0}$. Again substituting for $d \tilde{w}\left(w, \alpha, E_{0} ; E_{0}\right) / d E$, the RHS of (57) is

$$
\begin{aligned}
& \int_{\alpha_{E}^{0}}^{\alpha_{E}^{n+1}}\left(q_{E_{0}}(\alpha) \frac{\beta_{\theta}\left(E_{0}\right)}{E_{0}} w+\left(1-q_{E_{0}}(\alpha)\right) \frac{\beta_{\varphi}\left(E_{0}\right)}{E_{0}} w\right) q_{E_{0}}(\alpha) g_{E_{0}}(w, \alpha) d \alpha \\
& =\frac{\beta_{\theta}\left(E_{0}\right)}{E_{0}} w f_{E_{0}}^{\theta}(w)+\frac{\Delta\left(E_{0}\right)}{E_{0}} \int_{\alpha_{E}^{0}}^{\alpha_{E}^{n+1}} q_{E_{0}}(\alpha)\left(1-q_{E_{0}}(\alpha)\right) w g_{E_{0}}(w, \alpha) d \alpha
\end{aligned}
$$

Using the definitions in (53) and (54), we conclude that $d F_{E}^{\theta}(w) / d E=\frac{\beta_{\theta}(E)}{E} w f_{E}^{\theta}(w)-K_{E}(w)-L_{E}(w)$.

Finally, observe that $\tilde{q}_{E}(\alpha) \equiv q_{E}(\alpha)-\sum_{i=1}^{n}\left(\bar{q}^{i}-q^{i}\right) H\left(\alpha-\alpha_{E}^{i}\right)$, where $H$ is the Heaviside step function (using the half-maximum convention), is continuous. It has a well-defined derivative with respect to $E$ equal to $d q_{E}(\alpha) / d E$ away from the $\alpha_{E}^{i}$-jumps, and well-defined (bounded) left- and right-derivatives at the jumps. Therefore,

$$
\int_{\alpha=\alpha_{E}^{0}}^{\alpha_{E}^{n+1}} \int_{\underline{w}_{E}(\alpha)}^{w} \frac{d \tilde{q}_{E}(\alpha)}{d E} g_{E}\left(w^{\prime}, \alpha\right) d w^{\prime} d \alpha=\sum_{i=0}^{n} \int_{\alpha=\alpha_{E}^{i}}^{\alpha_{E}^{i+1}} \int_{\underline{w}_{E}(\alpha)}^{w} \frac{d q_{E}(\alpha)}{d E} g_{E}\left(w^{\prime}, \alpha\right) d w^{\prime} d \alpha .
$$


Differentiating $H$ as a distribution yields $\frac{d H\left(\alpha-\alpha_{E}^{i}\right)}{d E}=-\delta\left(\alpha-\alpha_{E}^{i}\right) \frac{d \alpha_{E}^{i}}{d E}$, where $\delta$ is the Dirac $\delta$-function. Hence,

$$
\int_{\alpha=\alpha_{E}^{0}}^{\alpha_{E}^{n+1}} \int_{\underline{w}_{E}(\alpha)}^{w}\left(\bar{q}^{i}-\underline{q}^{i}\right) \frac{d H\left(\alpha-\alpha_{E}^{i}\right)}{d E} g_{E}\left(w^{\prime}, \alpha\right) d w^{\prime} d \alpha=-\int_{\underline{w}_{E}\left(\alpha_{E}^{i}\right)}^{w} \frac{d \alpha_{E}^{i}}{d E}\left(\bar{q}^{i}-q^{i}\right) g_{E}\left(w^{\prime}, \alpha_{E}^{i}\right) d w^{\prime}
$$

Combining (58) and (59) (and differentiating $q_{E}(\alpha)$ as a distribution), we have

$\int_{\alpha=\alpha_{E}^{0}}^{\alpha_{E}^{n+1}} \int_{\underline{w}_{E}(\alpha)}^{w} \frac{d q_{E}(\alpha)}{d E} g_{E}\left(w^{\prime}, \alpha\right) d w^{\prime} d \alpha=\sum_{i=0}^{n} \int_{\alpha=\alpha_{E}^{i}}^{\alpha_{E}^{i+1}} \int_{\underline{w}_{E}(\alpha)}^{w} \frac{d q_{E}(\alpha)}{d E} g_{E}\left(w^{\prime}, \alpha\right) d w^{\prime} d \alpha-\sum_{i=0}^{n} \int_{\underline{w}_{E}\left(\alpha_{E}^{i}\right)}^{w} \frac{d \alpha_{E}^{i}}{d E}\left(\bar{q}^{i}-q^{i}\right) g_{E}\left(w^{\prime}, \alpha_{E}^{i}\right) d w^{\prime}$.

Using $Q\left(\zeta_{E}(\alpha)\right) \equiv q_{E}(\alpha)$ and $d \zeta_{E}(\alpha) / d E=\zeta_{E}(\alpha) \Delta(E) / E$ yields the second equality in (53).

Lemma 6 will be used in the now following proof of Lemma 5. Using (42) and a standard envelope theorem,

$$
\begin{aligned}
W^{\prime}(E) & =\int_{\underline{w}_{E}}^{\bar{w}_{E}} V(w) \frac{d \psi_{E}(w)}{d E} d w-\lambda \int_{\underline{w}_{E}}^{\bar{w}_{E}} c(V(w), l(w)) \frac{d f_{E}(w)}{d E} d w \\
& +\lambda(1-\xi) \int_{\underline{w}_{E}}^{\bar{w}_{E}} w l(w) \frac{d f_{E}^{\varphi}(w)}{d E} d w+\lambda \int_{\underline{w}_{E}}^{\bar{w}_{E}} w l(w) \frac{d f_{E}^{\theta}(w)}{d E} d w+\xi \lambda Y_{\varphi}^{\prime}(E)+B_{1}
\end{aligned}
$$

with

$$
\begin{array}{r}
B_{1} \equiv \quad \frac{d \bar{w}_{E}}{d E}\left[V\left(\bar{w}_{E}\right) \psi_{E}\left(\bar{w}_{E}\right)-\lambda c\left(V\left(\bar{w}_{E}\right), l\left(\bar{w}_{E}\right)\right) f_{E}\left(\bar{w}_{E}\right)+\lambda\left(f_{E}\left(\bar{w}_{E}\right)-\xi f_{E}^{\varphi}\left(\bar{w}_{E}\right)\right) \bar{w}_{E} e\left(\bar{w}_{E}\right)\right] \\
-\frac{d \underline{w}_{E}}{d E}\left[V\left(\underline{w}_{E}\right) \psi_{E}\left(\underline{w}_{E}\right)-\lambda c\left(V\left(\underline{w}_{E}\right), l\left(\underline{w}_{E}\right)\right) f_{E}\left(\underline{w}_{E}\right)+\lambda\left(f_{E}\left(\underline{w}_{E}\right)-\xi f_{E}^{\varphi}\left(\underline{w}_{E}\right)\right) \underline{w}_{E} e\left(\underline{w}_{E}\right)\right]
\end{array}
$$

and $Y_{\varphi}(E) \equiv r_{\varphi}(E) E$. Integrating by parts the four integrals yields

$$
\begin{aligned}
W^{\prime}(E)= & B_{1}+B_{2}-\int_{\underline{w}_{E}}^{\bar{w}_{E}} V^{\prime}(w) \frac{d \Psi_{E}(w)}{d E} d w+\lambda \int_{\underline{w}_{E}}^{\bar{w}_{E}}\left(\frac{V^{\prime}(w)}{u_{c}(w)}+\operatorname{MRS}(w) l^{\prime}(w)\right) \frac{d F_{E}(w)}{d E} d w \\
& -\lambda(1-\xi) \int_{\underline{w}_{E}}^{\bar{w}_{E}}\left(w l^{\prime}(w)+l(w)\right) \frac{d F_{E}^{\varphi}(w)}{d E} d w-\lambda \int_{\underline{w}_{E}}^{\bar{w}_{E}}\left(w l^{\prime}(w)+l(w)\right) \frac{d F_{E}^{\theta}(w)}{d E} d w+\xi \lambda Y_{\varphi}^{\prime}(E)
\end{aligned}
$$

with

$$
B_{2}=\left[V(w) \frac{d \Psi_{E}(w)}{d E}-\lambda c(V(w), l(w)) \frac{d F_{E}(w)}{d E}+\lambda(1-\xi) w l(w) \frac{d F_{E}^{\varphi}(w)}{d E}+\lambda w l(w) \frac{d F_{E}^{\theta}(w)}{d E}\right]_{\underline{w}_{E}}^{\bar{w}_{E}}
$$

By the first order conditions (46) and (48) with respect to $V(w)$ and $l(w)$ from the inner problem, the terms

$$
\begin{aligned}
& A_{1}(E) \equiv \lambda \int_{\underline{w}_{E}}^{\bar{w}_{E}} \frac{l^{\prime}(w)}{f_{E}(w)}\left[w f_{E}(w)\left(1-\frac{M R S(w)}{w}\right)-\xi w f_{E}^{\varphi}(w)-\eta(w)\left(\frac{\partial M R S(w)}{\partial l} \frac{l(w)}{w}+\frac{M R S(w)}{w}\right)\right] \frac{d F_{E}(w)}{d E} d w \\
& \text { and } A_{2}(E) \equiv \lambda \int_{\underline{w}_{E}}^{\bar{w}_{E}} \frac{V^{\prime}(w)}{u_{c}(w) f_{E}(w)}\left[\psi_{E}(w) \frac{u_{c}(w)}{\lambda}-f_{E}(w)-\eta^{\prime}(w)-\eta(w) \frac{\partial M R S(w)}{\partial c} l(w) \frac{y^{\prime}(w)}{y(w)}\right] \frac{d F_{E}(w)}{d E} d w
\end{aligned}
$$


are both equal to zero. Adding $A_{1}(E)$ and $A_{2}(E)$ to (61), using (13) and re-arranging yields

$$
\begin{aligned}
W^{\prime}(E)= & B_{1}+B_{2}+\xi \lambda Y_{\varphi}^{\prime}(E)+\int_{\underline{w}_{E}}^{\bar{w}_{E}} V^{\prime}(w)\left(\frac{\psi_{E}(w)}{f_{E}(w)} \frac{d F_{E}(w)}{d E}-\frac{d \Psi_{E}(w)}{d E}\right) d w \\
& -\lambda \int_{\underline{w}_{E}}^{\bar{w}_{E}} l(w) \frac{d F_{E}(w)}{d E} d w+\xi \lambda \int_{\underline{w}_{E}}^{\bar{w}_{E}}\left(\left(l(w)+w l^{\prime}(w)\right) \frac{d F_{E}^{\varphi}(w)}{d E}-w l^{\prime}(w) \frac{f_{E}^{\varphi}(w)}{f_{E}(w)} \frac{d F_{E}(w)}{d E}\right) d w \\
& -\lambda \int_{\underline{w}_{E}}^{\bar{w}_{E}}\left(\frac{\eta(w)}{w} \frac{d[M R S(w) l(w)]}{d w}+\eta^{\prime}(w) \frac{V^{\prime}(w)}{u_{c}(w)}\right) \frac{1}{f_{E}(w)} \frac{d F_{E}(w)}{d E} d w .
\end{aligned}
$$

Using Lemma 6, the first integral in (62) is

$$
\frac{\Delta(E)}{E} \int_{\underline{w}_{E}}^{\bar{w}_{E}} V^{\prime}(w) w \frac{f_{E}^{\theta}(w) f_{E}^{\varphi}(w)}{f_{E}(w)}\left(\frac{\psi_{E}^{\theta}(w)}{f_{E}^{\theta}(w)}-\frac{\psi_{E}^{\varphi}(w)}{f_{E}^{\varphi}(w)}\right) d w=\frac{\Delta(E)}{E} R
$$

Again using Lemma 6, the terms with $l(w)$ in the second line of (62) can be written as

$$
\begin{aligned}
-\lambda(1-\xi) \frac{\beta_{\varphi}(E)}{E} & \int_{\underline{w}_{E}}^{\bar{w}_{E}} w l(w) f_{E}^{\varphi}(w) d w-\lambda \frac{\beta_{\theta}(E)}{E} \int_{\underline{w}_{E}}^{\bar{w}_{E}} w l(w) f_{E}^{\theta}(w) d w+\xi \lambda \int_{\underline{w}_{E}}^{\bar{w}_{E}} l(w)\left(K_{E}(w)+L_{E}(w)\right) d w \\
& =-\lambda\left(\beta_{\varphi}(E) r_{\varphi}(E)+\beta_{\theta}(E) \frac{r_{\theta}(E)}{E} E_{\theta}\right)+\xi \lambda \beta_{\varphi}(E) r_{\varphi}(E)+\xi \lambda \int_{\underline{w}_{E}}^{\bar{w}_{E}} l(w)\left(K_{E}(w)+L_{E}(w)\right) d w \\
& =-\lambda r_{\varphi}(E) t_{\text {Pigou }}+\xi \lambda \beta_{\varphi}(E) r_{\varphi}(E)+\xi \lambda \int_{\underline{w}_{E}}^{\bar{w}_{E}} l(w)\left(K_{E}(w)+L_{E}(w)\right) d w
\end{aligned}
$$

The terms with $w l^{\prime}(w)$ in (62) can be written as

$$
\begin{aligned}
& \xi \lambda \int_{\underline{w}_{E}}^{\bar{w}_{E}} w l^{\prime}(w)\left[\frac{\beta_{\varphi}(E)}{E} w f_{E}^{\varphi}(w)-\frac{f_{E}^{\varphi}(w)}{f_{E}(w)} \frac{w}{E}\left(\beta_{\varphi}(E) f_{E}^{\varphi}(w)+\beta_{\theta}(E) f_{E}^{\theta}(w)\right)+K_{E}(w)+L_{E}(w)\right] d w \\
= & \xi \lambda \frac{\Delta(E)}{E} \int_{\underline{w}_{E}}^{\bar{w}_{E}} w^{2} l^{\prime}(w)\left[\frac{f_{E}^{\theta}(w) f_{E}^{\varphi}(w)}{f_{E}(w)}-\int_{0}^{\infty} q_{E}(\alpha)\left(1-q_{E}(\alpha)\right) g_{E}(w, \alpha) d \alpha\right] d w+\xi \lambda \int_{\underline{w}_{E}}^{\bar{w}_{E}} w l^{\prime}(w) K_{E}(w) d w \\
= & \xi \lambda \frac{\Delta(E)}{E} \int_{\underline{w}_{E}}^{\bar{w}_{E}} w^{2} l^{\prime}(w) \operatorname{Var}_{E}(q \mid w) f_{E}(w) d w+\xi \lambda \int_{\underline{w}_{E}}^{\bar{w}_{E}} w l^{\prime}(w) K_{E}(w) d w \\
= & \xi \lambda \frac{\Delta(E)}{E} C+\xi \lambda \int_{\underline{w}_{E}}^{\bar{w}_{E}} w l^{\prime}(w) K_{E}(w) d w
\end{aligned}
$$

where the first equality uses (54). Combining the terms with $K_{E}(w)$ from (64) and (65) gives $\xi \lambda \int_{\underline{w}_{E}}^{\bar{w}_{E}}(w l(w))^{\prime} K_{E}(w) d w$, which can be integrated by parts to yield

$$
B_{3}-\xi \lambda \int_{\underline{w}_{E}}^{\bar{w}_{E}} w l(w) K_{E}^{\prime}(w) d w=B_{3}+\xi \lambda \frac{\Delta(E)}{E} \int_{\underline{w}_{E}}^{\bar{w}_{E}} \int_{0}^{\infty} y(w) Q^{\prime}\left(\zeta_{E}(\alpha)\right) \zeta_{E}(\alpha) d G_{E}(w, \alpha)
$$

with $B_{3}=\xi \lambda \bar{w}_{E} l\left(\bar{w}_{E}\right) K_{E}\left(\bar{w}_{E}\right)$ since $K_{E}\left(\underline{w}_{E}\right)=0$. Further combining this with the $L_{E}(w)$-term in (64) yields

$$
B_{3}+\xi \lambda \frac{\Delta(E)}{E} \int_{\underline{w}_{E}}^{\bar{w}_{E}} y(w) \int_{0}^{\infty}\left(Q^{\prime}\left(\zeta_{E}(\alpha)\right) \zeta_{E}(\alpha)-q_{E}(\alpha)\left(1-q_{E}(\alpha)\right)\right) d G_{E}(w, \alpha) .
$$


Note that $\tilde{Q}^{\prime}(\zeta)=\left.\tilde{Q}_{\zeta_{0}}^{\prime}(\zeta)\right|_{\zeta_{0}=\zeta}=Q^{\prime}(\zeta) \zeta-Q(\zeta)(1-Q(\zeta))$, so that $(67)$ becomes

$$
B_{3}+\xi \lambda \frac{\Delta(E)}{E} \int_{\underline{w}_{E}}^{\bar{w}_{E}} \int_{0}^{\infty} y(w) \tilde{Q}^{\prime}\left(\zeta_{E}(\alpha)\right) \zeta_{E}(\alpha) d G_{E}(w, \alpha)=B_{3}+\xi \lambda \frac{\Delta(E)}{E} S .
$$

Moreover, $S \geq 0$ since $\tilde{Q}^{\prime}(\zeta)=\zeta^{2} x^{\prime}(\zeta) /(1+\zeta x(\zeta))^{2} \geq 0$. Finally, use the incentive constraint (13), rewritten as $V^{\prime}(w) / u_{c}(w)=\operatorname{MRS}(w) l(w) / w$, to write the last line of (62) as

$$
-\lambda \int_{\underline{w}_{E}}^{\bar{w}_{E}}\left(\eta(w) w \frac{d\left[V^{\prime}(w) / u_{c}(w)\right]}{d w}+\eta^{\prime}(w) w \frac{V^{\prime}(w)}{u_{c}(w)}+\eta(w) \frac{V^{\prime}(w)}{u_{c}(w)}\right) \frac{1}{w f_{E}(w)} \frac{d F_{E}(w)}{d E} d w
$$

or, recognizing the sum of the bracketed terms as $d\left[\eta(w) w V^{\prime}(w) / u_{c}(w)\right] / d w$, integrating by parts, and using the transversality condition $\eta\left(\underline{w}_{E}\right)=\eta\left(\bar{w}_{E}\right)=0$ and (52),

$$
\begin{aligned}
& \lambda \int_{\underline{w}_{E}}^{\bar{w}_{E}} \eta(w) w \frac{V^{\prime}(w)}{u_{c}(w)} \frac{d}{d w}\left(\frac{\beta_{\theta}(E)}{E} \frac{f_{E}^{\theta}(w)}{f_{E}(w)}+\frac{\beta_{\varphi}(E)}{E} \frac{f_{E}^{\varphi}(w)}{f_{E}(w)}\right) d w \\
&=\lambda \frac{\Delta(E)}{E} \int_{\underline{w}_{E}}^{\bar{w}_{E}} \eta(w) w \frac{V^{\prime}(w)}{u_{c}(w)} \frac{d}{d w}\left(\frac{f_{E}^{\varphi}(w)}{f_{E}(w)}\right) d w=\frac{\Delta(E)}{E} I .
\end{aligned}
$$

Define $\tilde{F}(w, E) \equiv F_{E}(w)$. Since $\tilde{F}\left(\bar{w}_{E}, E\right) \equiv 1$ for all $E$,

$$
\frac{d \tilde{F}\left(\bar{w}_{E}, E\right)}{d E}=\frac{\partial \tilde{F}\left(\bar{w}_{E}, E\right)}{\partial E}+\frac{\partial \tilde{F}\left(\bar{w}_{E}, E\right)}{\partial w} \frac{d \bar{w}_{E}}{d E}=\frac{d F_{E}\left(\bar{w}_{E}\right)}{d E}+f_{E}\left(\bar{w}_{E}\right) \frac{d \bar{w}_{E}}{d E}=0 .
$$

Together with an analogous expression at $\underline{w}_{E}$ and the fact that $K_{E}\left(\underline{w}_{E}\right)=L_{E}\left(\underline{w}_{E}\right)=L_{E}\left(\bar{w}_{E}\right)=0$, this yields $B_{1}+B_{2}=-\xi \lambda \bar{w}_{E} l\left(\bar{w}_{E}\right) K_{E}\left(\bar{w}_{E}\right)=-B_{3}$. Using (63), (64), (65), (68) and (69) in (62) yields

$$
W^{\prime}(E)=-\lambda r_{\varphi}(E) t_{\text {Pigou }}+\frac{\Delta(E)}{E}(R+I)+\xi \lambda\left(r_{\varphi}(E)+\frac{\Delta(E)}{E}(C+S)\right),
$$

where we have used $Y_{\varphi}^{\prime}(E)+\beta_{\varphi}(E) r_{\varphi}(E)=r_{\varphi}(E)$. This completes the proof of Lemma 5 .

To see that Lemma 3 is implied as a special case when $m$ is linear, observe that then $n=1, \bar{q}^{1}=1$, $\underline{q}^{1}=0, s_{1}=1$, and $\alpha_{E}^{1}=\frac{r_{\varphi}(E)}{r_{\theta}(E)}$. Hence, for the region where $q=1$,

$$
K_{E}(w)=\sum_{i=1}^{n} \int_{\underline{w}_{E}}^{w} \frac{d \alpha_{E}^{i}}{d E}\left(\bar{q}^{i}-\underline{q}^{i}\right) g_{E}\left(w^{\prime}, \alpha_{E}^{i}\right) d w^{\prime}=\frac{d}{d E}\left(\frac{r_{\varphi}(E)}{r_{\theta}(E)}\right) \int_{\underline{w}_{E}}^{w} g_{E}\left(w^{\prime}, \frac{r_{\varphi}(E)}{r_{\theta}(E)}\right) d w^{\prime}
$$

In this region, $w=\theta r_{\theta}(E)$ and $\alpha=\theta / \varphi$. Hence, $\theta=w / r_{\theta}(E), \varphi=\frac{w}{\alpha r_{\theta}(E)}$ and the Jacobian for the transformation is $\frac{w}{\alpha^{2} r_{\theta}(E)^{2}}$. Therefore, $g_{E}\left(w, \frac{r_{\varphi}(E)}{r_{\theta}(E)}\right)=\frac{w}{\left(\alpha_{E}^{1}\right)^{2} r_{\theta}(E)^{2}} f\left(\frac{w}{r_{\theta}(E)}, \frac{w}{\alpha_{E}^{1} r_{\theta}(E)}\right)$, or, transforming to $\varphi=$ $w / r_{\varphi}(E)$,

$$
K_{E}(w)=\frac{d}{d E}\left(\frac{r_{\varphi}(E)}{r_{\theta}(E)}\right) \int_{\underline{w}_{E}}^{w} g_{E}\left(w^{\prime}, \frac{r_{\varphi}(E)}{r_{\theta}(E)}\right) d w^{\prime}=-\frac{\Delta(E)}{E} \frac{r_{\varphi}(E)}{r_{\theta}(E)} \int_{\underline{\varphi}}^{w / r_{\varphi}(E)} \varphi f\left(\varphi \frac{r_{\varphi}(E)}{r_{\theta}(E)}, \varphi\right) d \varphi .
$$

Moreover, $L_{E}(w)=0$ since $q \in\{0,1\}$. Using this in (65) and (66) yields the result. 


\section{C.4 Proof of Proposition 3}

The first part of the proposition is established by the following lemma:

Lemma 7. $\xi>0$ in any regular Pareto optimum.

Proof. Any Pareto optimum solves $\max _{E, T(.)} \int_{\underline{w}_{E}}^{\bar{w}_{E}} u\left(y_{T}(w)-T\left(y_{T}(w)\right), y_{T}(w) / w\right) d \Psi_{E}(w)$ subject to (a) a set of incentive constraints $y_{T}(w) \in \arg \max _{y} u(y-T(y), y / w)$ for all $w$, (b) the consistency constraint $Y_{\varphi}(E)-\int_{\underline{w}_{E}}^{\bar{w}_{E}} y_{T}(w) f_{E}^{\varphi}(w) d w=0$ and (c) the revenue constraint $\int_{\underline{w}_{E}}^{\bar{w}_{E}} T\left(y_{T}(w)\right) d F_{E}(w) \geq 0$. We show that any solution to the relaxed problem where the consistency constraint (c) is replaced by the inequality constraint $Y_{\varphi}(E)-\int_{\underline{w}_{E}}^{\bar{w}_{E}} y_{T}(w) f_{E}^{\varphi}(w) d w \geq 0$, with associated multiplier $\lambda \tilde{\xi}$, always has $\tilde{\xi}>0$ with regular $\Psi$. Since this implies that the solution $\left(E^{*}, T^{*}().\right)$ to the relaxed problem is feasible in the unrelaxed problem, $\xi=\tilde{\xi}>0$ as well. So, suppose, by way of contradiction, that $\tilde{\xi}=0$ in $\left(E^{*}, T^{*}().\right)$. Standard arguments (e.g. Werning, 2000) imply $T^{*^{\prime}}() \geq$.0 with regular Pareto weights in this case. Now consider a small decrease $\Delta E$ from $E^{*}$ holding $T^{*}$ (.) fixed. This at least weakly increases the wage, and hence the utility, of each individual, increasing the objective. It has no effect on the set of incentive constraints (a) since $T^{*}($. remains fixed. It has no effect on the relaxed constraint $(b)$ since $\tilde{\xi}=0$. It relaxes the revenue constraint (c) since $y_{T}(w)$ is non-decreasing and $T^{*^{\prime}}() \geq$.0 . This contradicts the optimality of $\left(E^{*}, T^{*}().\right)$ in the relaxed problem, showing that $\tilde{\xi}>0$ in the relaxed problem and hence $\tilde{\xi}=\tilde{\xi}>0$.

We next show that $\eta(w) \geq 0$ under the assumptions in the proposition. To see this, suppose (by way of contradiction) $\eta(w)<0$ for some $w$. Since $\eta\left(\underline{w}_{E}\right)=\eta\left(\bar{w}_{E}\right)=0$ by the transversality condition, this together with continuity of $\eta(w)$ implies that there exists some interval $\left[w_{1}, w_{2}\right]$ such that $w_{1}<w_{2}, \eta\left(w_{1}\right)=\eta\left(w_{2}\right)=$ 0 and $\eta(w)<0$ for all $w \in\left(w_{1}, w_{2}\right)$. Then $\eta^{\prime}\left(w_{1}\right) \leq 0$ and $\eta^{\prime}\left(w_{2}\right) \geq 0$. Using (18), this implies

$$
\frac{\psi_{E}\left(w_{1}\right)}{f_{E}\left(w_{1}\right)} \frac{u_{c}\left(w_{1}\right)}{\lambda} \leq \frac{\psi_{E}\left(w_{2}\right)}{f_{E}\left(w_{2}\right)} \frac{u_{c}\left(w_{2}\right)}{\lambda} .
$$

$\psi_{E}(w) / f_{E}(w)$ is decreasing in $w$ with regular Pareto weights and $u_{c}(w)$ is also decreasing under condition (ii), yielding the desired contradiction. Hence, $I$ is non-negative under condition (iii). Conditions (i) and (iv) ensure that $C$ and $R$ are also non-negative, and $S>0$. Hence, either the numerator or the denominator of (24) or both are positive. $\xi>0$ implies that both are positive. Hence, $\xi \lesseqgtr t_{\text {Pigou }} \Leftrightarrow \Delta(E) \gtreqless 0$.

\section{Appendix: Details on Section 5}

\section{D.1 Data and Estimation}

Data. We use the March release of the CPS for 2014. We use the earnings data and the self-reported estimate of hours worked to construct wages. The CPS also provides a detailed industry classification for working individuals. We drop individuals for whom earnings, hours, age or industry is not reported. Following Heathcote et al. (2010), we also restrict attention to working age individuals between ages 25 and 65 and those employed (dropping those with very low hours or earnings per year). All variables are weighted with the provided weights.

The industry variable we use to classify individuals, for the sake of our illustration, as rent-seekers or traditional workers is a 3-digit NAICS-based industry code. This is the variable ind02 in the NBER released Merged Outgoing Rotation Groups (MORG) data. We choose the finance and law-related categories "Banking and related activities," "Savings institutions, including credit unions," "Non-depository credit 

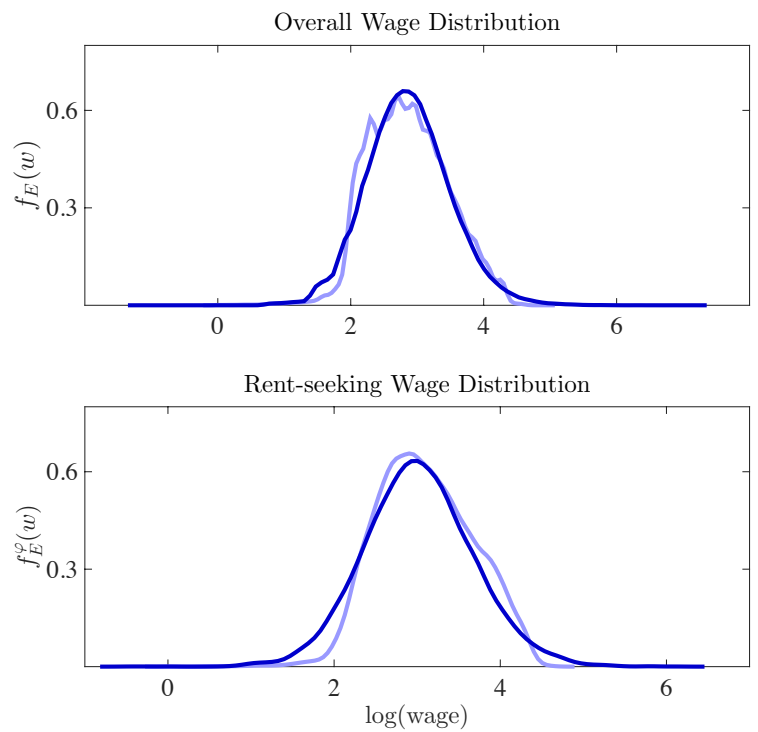
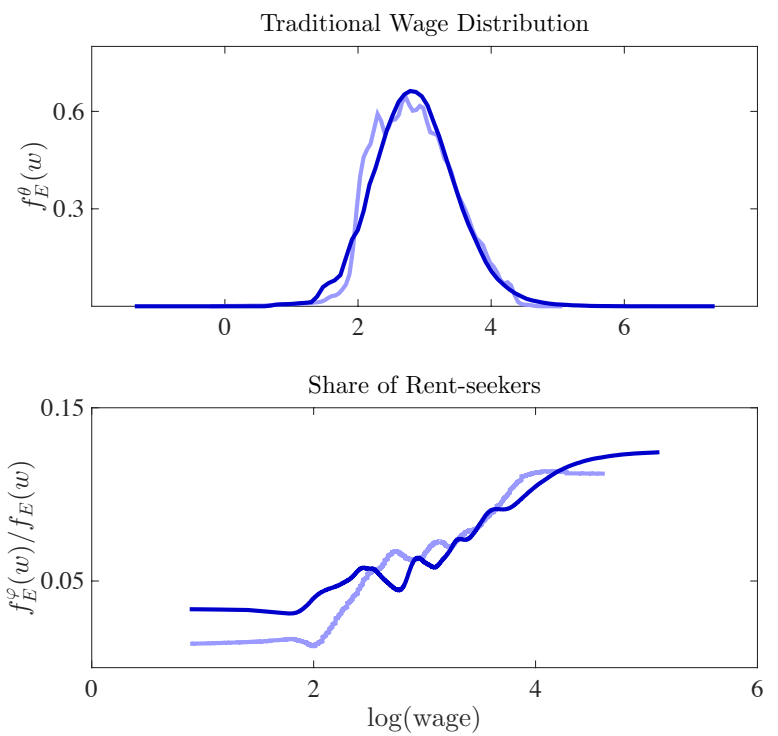

Figure 2: Empirical and fitted (sectoral) log-wage distributions

and related activities," "Securities, commodities, funds, trusts, and other financial investments," "Insurance carriers and related activities," and "Legal services" to capture rent-seeking (following the spirit of, and simplifying comparison to, Lockwood et al., 2015).

Normalizations. From the data, we obtain the (per-capita) incomes of traditional workers and rent-seekers, $Y_{\theta}$ and $Y_{\varphi}$. We can, w.l.o.g., normalize $E_{\theta}=Y_{\theta}$ and $E=Y_{\varphi}$ in this initial allocation, so that $r_{\theta}=Y_{\theta} / E_{\theta}=1$ and $r_{\varphi}=Y_{\varphi} / E=1$ and wages coincide with skills. This is because scaling all $\theta$-skills by some factor $k$ scales $E_{\theta}$ by $k$ and hence $r_{\theta}$ by $1 / k$, which leaves traditional wages $w_{\theta}=\theta r_{\theta}$, efforts and incomes unchanged. The same holds for $\varphi$-skills. In other words, skills $(\theta, \varphi)$ are only identified up to such re-scalings for given observables. Given our specification, $r_{\theta}=k_{\theta} E^{-\beta_{\theta}}$ and $r_{\varphi}=k_{\varphi} E^{-\beta_{\varphi}}$, so this normalization implies setting the constants $k_{\theta}$ and $k_{\varphi}$ such that $k_{\theta}=Y_{\varphi}^{\beta_{\theta}}$ and $k_{\varphi}=Y_{\varphi}^{\beta_{\varphi}}$.

Estimation. Let $\left(w_{\theta i}, w_{\varphi i}\right)$ be individual $i$ 's potential wages in the traditional and rent-seeking activities, where the observed wage in the chosen sector is $w_{i}=\max \left\{w_{\theta i}, w_{\varphi i}\right\}$. Our goal is to estimate the 7 parameters of the bivariate Pareto-lognormal wage distribution described in Section 5 in order to approximate the empirical sectoral wage distributions from the data. We use a standard GMM estimation procedure to accomplish this, based on the following moment conditions. We first compute $N$ equal quantiles of the overall empirical wage distribution, taking both sectors together, and then find the share of individuals in each of these quantiles and in each of the two sectors. This gives us $2 N$ moments to match, of which 1 is redundant and therefore dropped (since the shares add up to 1). We work with $N=100$ (i.e., percentiles), which helps to match all parts of the sectoral wage distributions, including the top tail, but also perform robustness checks with fewer quantiles, for instance the $N=20$ or $N=5$, with relatively minor effects on the estimation results. ${ }^{34}$

\footnotetext{
${ }^{34}$ These are standard moment conditions of the form $\mathbb{E}\left[g\left(w_{i}, s_{i}, \pi\right)\right]=0$, where $g\left(w_{i}, s_{i}, \pi\right)=v\left(w_{i}, s_{i}\right)-$ $\bar{v}(\pi)$ is the difference between the $2 N$ - 1-dimensional vector $v\left(w_{i}, s_{i}\right)$ with each element equal to an indicator for the quantile/sector of observation $i$ (implied by the wage $w_{i}$ and sector $s_{i}$ ) and the $2 N-1$ -
} 

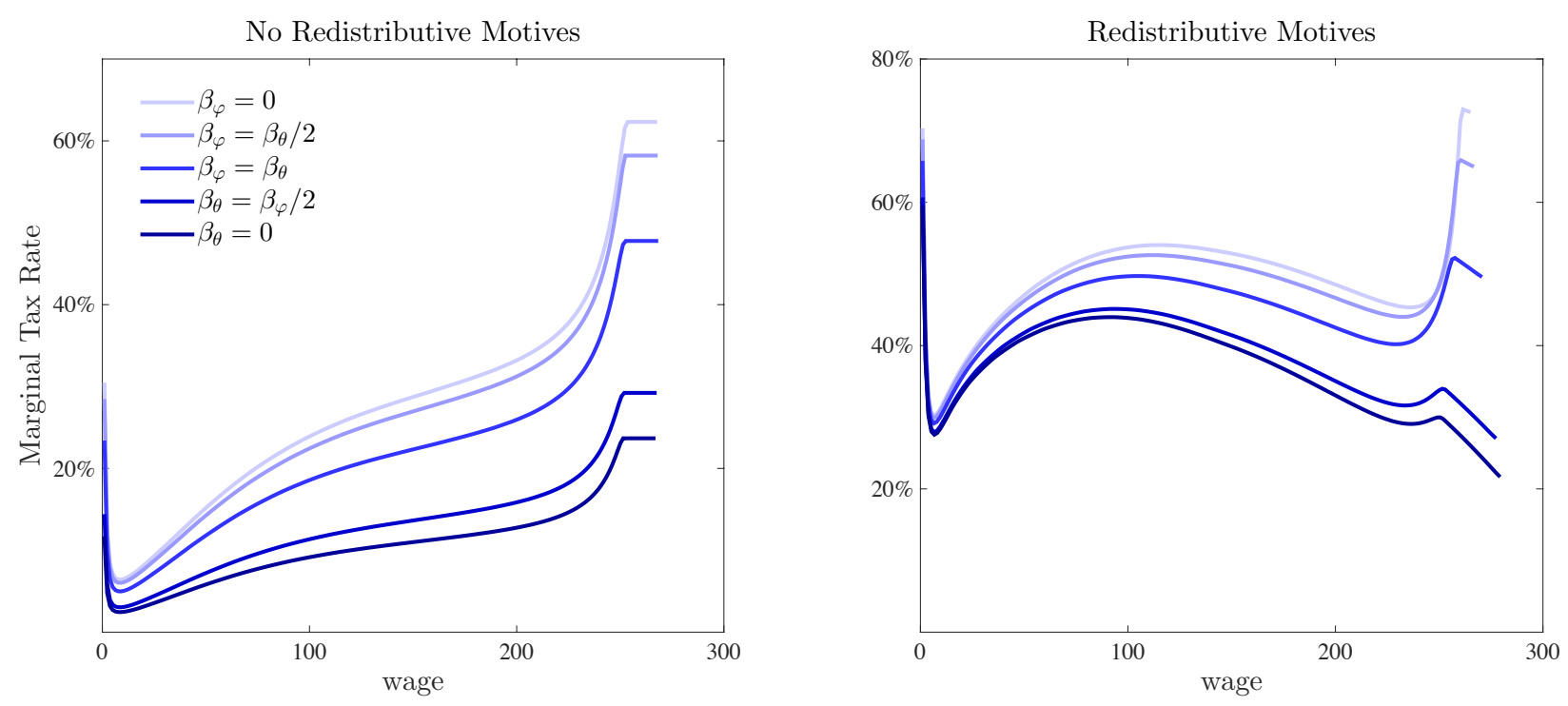

Figure 3: Optimal policy $t_{\text {Pigou }}=50 \%$, various scenarios

We use a standard two-step estimator. In the first step, we obtain a consistent (albeit not efficient) parameter estimate by numerically minimizing the unweighted sum of squared differences between the empirical shares and the shares under the parametric distribution. Using this parameter estimate, we can repeatedly simulate the shares in each quantile/sector to obtain a consistent estimate of their covariance matrix. Second, we re-estimate using the inverse of this covariance matrix as a weighting matrix. We obtain $\mu_{\theta}=2.5, \sigma_{\theta}=0.5, \alpha_{\theta}=3.7, \mu_{\varphi}=1.7, \sigma_{\varphi}=0.8, \alpha_{\varphi}=3.2$ and $\rho=0.6$.

Fit. Figure 2 plots the resulting distributions of log-wages (both overall and for the two sectors) as well as the share of rent-seekers as a function of the log-wage, both for the data and the fitted bivariate Paretolognormal, which demonstrates a reasonably good fit. In particular, we find a lower Pareto-parameter (and hence a thicker tail) in the $\varphi$-dimension, which implies that rent-seekers dominate at the very top of the wage distribution. We truncate the resulting skill distribution at the top 0.1 percentile in both dimensions and rescale accordingly.

Policy Simulations. To compute optimal income taxes, we begin with the inner problem for given $E$. The (sectoral) wage distributions are obtained numerically using the return functions $r_{i}(E)$ and the estimated skill distribution. The resulting optimal tax problem can be solved using the same methods as for a standard Mirrlees model (making use of the local incentive constraints), with the only additional complication that the multiplier $\xi$ needs to be found numerically such that the consistency constraint is satisfied. We then repeat this procedure to find the optimal $E$ using a grid search. We finally verify that the monotonicity constraint $y^{\prime}(w) \geq 0$ is satisfied, so the solution is globally incentive compatible.

dimensional vector $\bar{v}(\pi)$ of quantile/sector shares under the bivariate Pareto-lognormal wage distribution with 7-dimensional parameter vector $\pi$. Our moment condition-the difference between the observed fraction of individuals in each bin and the expected fraction given $\pi$-is the empirical analog of $\mathbb{E}\left[g\left(w_{i}, s_{i}, \pi\right)\right]$. 
Table 2: Optimal $\xi$ for different redistributive and technological scenarios

\begin{tabular}{lccccc}
\hline & $\beta_{\theta}=0$ & $\beta_{\theta}=\beta_{\varphi} / 2$ & $\beta_{\theta}=\beta_{\varphi}$ & $\beta_{\varphi}=\beta_{\theta} / 2$ & $\beta_{\varphi}=0$ \\
\hline No redistribution & $23.7 \%$ & $29.2 \%$ & $50.6 \%$ & $58.2 \%$ & $62.3 \%$ \\
Redistribution & $21.6 \%$ & $27.0 \%$ & $52.0 \%$ & $65.0 \%$ & $72.6 \%$ \\
\hline
\end{tabular}

Sensitivity Checks. In Section 5, we compute optimal tax policy for an economy calibrated such that $t_{\text {Pigou }}=25 \%$. Here we show how the results change for twice as strong negative externalities, i.e. $t_{\text {Pigou }}=$ $50 \%$. Figure 3 plots the resulting marginal tax rate schedules, as a function of the wage, for all 5 scenarios capturing possible relative return effects as well as without and with redistributive motives $(r=1$ versus $r=1.3$, left versus right panel). Table 2 lists the corresponding optimal corrections $\xi$. See Section 5 for a brief discussion. 